Article

\title{
Physically Acceptable Embedded Class-I Compact Stars in Modified Gravity with Karmarkar Condition
}

\author{
Saira Waheed ${ }^{1, *}$, Ghulam Mustafa ${ }^{2}$, Muhammad Zubair ${ }^{3}$ and Asifa Ashraf ${ }^{4}$ \\ 1 Faculty of CORE Humanities, Prince Mohammad Bin Fahd University, Al Khobar 31952, Saudi Arabia \\ 2 Department of Mathematics, Shanghai University, Shanghai 200444, China; gmustafa3828@gmail.com \\ 3 Department of Mathematics, COMSATS University Islamabad, Lahore Campus, Punjab 5459, Pakistan; \\ drmzubair@cuilahore.edu.pk \\ 4 Jiangsu Key Labortary for NSLSCS, School of Mathematical Sciences, Nanjing Normal University, \\ Nanjing 210023, China; asifa.ashraf70@yahoo.com \\ * Correspondence: swaheed@pmu.edu.sa
}

Received: 27 March 2020; Accepted: 7 May 2020 ; Published: 5 June 2020

\begin{abstract}
The present study is devoted to explore the existence of a new family of compact star solutions by adopting the Karmarkar as well as Pandey-Sharma condition in the background of $f(\mathscr{R}, \mathcal{T})$ modified gravitational framework. For this purpose, we consider static spherically symmetric spacetime with anisotropic fluid distribution in absence of electric charge. In respect of Karmarkar condition, we assume a specific model of $g_{r r}$ metric potential representing a new family of solutions which is also compatible with the Pandey-Sharma condition. This assumed model permits us to calculate the $g_{t t}$ component of metric tensor by making the use of Karmarkar condition. Further, we investigate the interior solutions for Vela $X-1$ model of compact star by utilizing this new family of solutions for different values of parameter $\lambda$. We have tuned the solution for Vela $X-1$ so that the solutions matches the observed mass and radius. For the same star we have extensively discussed the behavior of the solutions. It is found that these solutions fulfill all the necessary conditions under the observational radii and mass attribute data for small values of parameter $\lambda$ and hence physically well-behaved and promising. Through graphical analysis, it is observed that our obtained analytical solutions are physically acceptable with a best degree of accuracy for $n \in[1.8,7)-\{2,4,6\}$, where parameter $n$ is involved in the discussed model. It is also noticed the causality condition is violated for all $n \geq 7$ and the tangential sound velocity $v_{t}$ is observed as complex valued for all $0<n<1$. Likewise, we explore these properties by considering large parameter $\lambda$ values. It is seen that the presented model violates all the physical conditions for $n \in\{2,4,6\}$, while some of these for large values of $\lambda$. Consequently, it can be concluded that the parameters $n$ and $\lambda$ have a strong impact on the obtained solutions.
\end{abstract}

Keywords: karmarkar condition; $f(\mathscr{R}, \mathcal{T})$ theory; compact stars

\section{Introduction}

The study of relativistic compact stars in the background of general relativity (GR) and extended theories of gravity is considered as one of the most promising subjects among the researchers during last few decades. The exact solution of Einstein's field equations (EFEs) for the first time was calculated by Schwarzschild [1] in 1916 for the interior of a compact object. Later various researchers made efforts to obtain the new exact solutions of EFEs in reference to different astrophysical and cosmological aspects. Tolman [2] and Oppenheimer [3] both studied some more realistic and convoluted models of non-traversable relativistic objects in proximity with data-based actuality. In 1939, Oppenheimer and Snyder [4] examined the phenomenon of gravitational collapse with homogeneity based dust 
sphere and they presented an interesting and simple collapse mode in this respect. In 1922, Jeans [5] introduced the anisotropic pressure in self gravitational bodies in the context of Newtonian gravity. Further, in 1933, Lemaitre [6] used the anisotropic model for the first time in the background of GR by taking constant energy density with a radial pressure source. Later on, Bowers and Liang [7], in 1974, employed anisotropic spheres in GR. Ruderman [8] argued that the existence of nonzero anisotropy is regarded as a significant component of a stellar object without an electric charge. Gleiser and Dev [9] argued that pressure anisotropy impacts the structure, mass and other physical properties of extremely compact spheres. Herrera and Santos [10] described the effect of anisotropy on the characteristics of the stellar stars. The MIT bag model arising from observations in fundamental particle physics relates the pressure to the density of the star via a linear relation of the form $p=\alpha \rho-B$ where $B$ is the Bag constant. This equation of state has been successfully used to model compact objects in general relativity ranging from neutron stars through to strange star candidates.

The most interesting fact of modern cosmology is the accelerated expanding nature of our cosmos which is also supported by the suggestions coming from different recent observational experiments like LSS, CMB, BAO and Supernovae-Ia [11-14]. It is argued that the standard GR theory fails to describe the hidden exotic nature of its responsible factor labeled as dark energy (DE). It is realized that exotic cosmological astral structures may be determined by reviewing the relativistic astral objects in the framework of modified theory of gravity. It is also argued that recent data from solar system tests of gravity might also be compatible with some other theories of gravity $[15,16]$. In the literature, many alternative theories of gravity are available including $f(\mathscr{R})$ theory, $f(T)$ gravity, $f(\mathrm{G})$ theory, $f\left(T, T_{G}\right)$ modified framework, braneworld scenario, Gauss-Bonnet gravity, Kalb-Ramond background and scalar-tensor theories especially Brans-Dicke gravity [16-23]. It is argued that GR theory faced stringent constraints for describing the galactic scale, the strong lensing regime and CMBR tests. In this respect, its recently proposed extended version namely $f(\mathscr{R})$ theory is considered as quite successful and interesting because of its correspondence to scalar-tensor gravity, i.e., the GR with an extra scalar-field candidate, whose scalar-potential is in double correspondence to the generic function $f$ [24].

Recently, another interesting modification of $f(\mathscr{R})$ theory has been proposed in the literature [25] by taking an arbitrary function into account namely $f(\mathscr{R}, \mathcal{T})$, where $\mathcal{T}$ represents the trace of energy-momentum matter of the ordinary matter lagrangian part of the action. This modification is acknowledged as $f(\mathscr{R}, \mathcal{T})$ modified theory. The $f(\mathscr{R}, \mathcal{T})$ theory has drawn much attention of the investigators and many interesting results have been found in the cosmological aspects [26-30]. Sharif et al. [31] explored $f(\mathscr{R}, \mathcal{T})$ theory of gravity to investigate some features related to the stability of a locally isotropic self-gravitating system. Zubair et al. [32] discussed the possible formation of compact objects by employing the Krori and Barua metric in the background of $f(\mathscr{R}, \mathcal{T})$ theory. Alhamzawi and Alhamzawi [33] described the phenomenon of gravitational lensing in the framework of $f(\mathscr{R}, \mathcal{T})$ theory. The topic of stability has been investigated numerically by Moraes et al. [34] using TOV equation in $f(\mathscr{R}, \mathcal{T})$ theory. Das et al. [35] calculated an exact solution of compact objects using modified field equations by taking the conformal symmetry motion into account in the background of $f(\mathscr{R}, \mathcal{T})$ theory.

In the literature, numerous researchers have recommended an embedding spacetime metric for the basic structure of compact stars which is consistent with Karmarkar condition defined by $\mathscr{R}_{1414}=\left(\mathscr{R}_{1212} \mathscr{R}_{3434}+\mathscr{R}_{1224} \mathscr{R}_{1334}\right) / \mathscr{R}_{2323}$ [36]. The Karmarkar condition provides a connection between the two main parts of metric potentials of a spherically symmetric spacetime. By adopting Karmarkar condition, one of the metric components can be calculated which is further capable of generating the second metric component [37-39]. In this respect, various families of solutions are generated by Bhar et al. [40,41], Maurya et al. [42-44] and Newton Singh et al. [45-48] which are physically acceptable for embedding spacetimes and can be used to describe the interior solution of stellar objects. These new families of solutions satisfying the EFEs along with Karmarkar condition are termed as embedding class-I solutions. Further, many different stellar models have been assumed 
to investigate the new well-behaved classes of solutions by considering the connection of metric potentials in embedding class-I solutions [49-51]. Recently, Mustafa et al. [51,52] have explored the realistic stellar anisotropic model with well behaved nature solutions satisfying Karmarker condition in $f(\mathscr{R}, \mathcal{T})$ gravity.

The present study investigates the existence of spherical symmetric solutions for Vela $X-1$ model of compact stars in the framework of $f(\mathscr{R}, \mathcal{T})$ theory of gravity. For this purpose, we shall adopt the Karmarkar as well as Pandey Sharma condition for simplification purposes. The paper is designed in the following order of sections. In the next section, we shall present the mathematical formulations of modified $f(\mathscr{R}, \mathcal{T})$ theory of gravity along with its field equations for a spherically symmetric geometry with anisotropic matter source. Here we shall discuss a new configuration of embedding class-I solutions in $f(\mathscr{R}, \mathcal{T})$ theory and the physical boundary conditions in the same section. Section 3 will provide a detailed graphical analysis of some physical properties and the stability of Vela $X-1$ model of compact stars for different values of $\lambda$. Final section concludes the whole work by focusing on major findings.

\section{Basic Structure of $f(\mathscr{R}, \mathcal{T})$ Gravitational Framework}

In this part, we shall explain the essential structure of modified $f(\mathscr{R}, \mathcal{T})$ gravitational framework with all the necessary assumptions taken for this work. The action of $f(\mathscr{R}, \mathcal{T})$ modified theory is defined by [24]:

$$
\mathcal{S}=\frac{1}{16 \pi} \int f(\mathscr{R}, \mathcal{T}) \sqrt{-g} d^{4} x+\int \mathcal{L}_{\mathrm{m}} \sqrt{-g} d^{4} x,
$$

where $\mathcal{L}_{\mathrm{m}}$ symbolizes the ordinary matter Lagrangian density. By taking variation of the above action in Equation (1), we get the following set of dynamical equations

$$
8 \pi \mathcal{T}_{\mu v}-f_{\mathcal{T}}(\mathscr{R}, \mathcal{T}) \mathcal{T}_{\mu v}-f_{\mathcal{T}}(\mathscr{R}, \mathcal{T}) \Theta_{\mu v}=-\frac{1}{2} f(\mathscr{R}, \mathcal{T}) g_{\mu v}+f_{\mathscr{R}}(\mathscr{R}, \mathcal{T}) \mathscr{R}_{\mu v}+\left(g_{\mu v} \square-\nabla_{\mu} \nabla_{v}\right) f_{\mathscr{R}}(\mathscr{R}, \mathcal{T}) .
$$

In this set of field equations, the notations $\nabla$ and $\square$ are, respectively introduced for the covariant derivative and the d'Alembert operator defined by $\square=\partial_{\mu}\left(\sqrt{-g} g^{\mu v} \partial_{v}\right) / \sqrt{-g}$. Further, the function $f$ with subscripts refer to the derivatives given by $f_{\mathscr{R}}(\mathscr{R}, \mathcal{T})=\partial f(\mathscr{R}, \mathcal{T}) / \partial \mathscr{R}$ and $f_{T}(\mathscr{R}, \mathcal{T})=\partial f(\mathscr{R}, \mathcal{T}) / \partial \mathcal{T}$. In addition, the term $\Theta_{\mu v}$ is defined by

$$
\Theta_{\mu v}=\frac{g^{\alpha \beta} \delta \mathcal{T}_{\mu v}}{\delta g^{\mu \nu}}=-2 \mathcal{T}_{\mu v}+g_{\mu \nu} \mathcal{L}_{\mathrm{m}}-2 g^{\alpha \beta} \frac{\partial^{2} \mathcal{L}_{\mathrm{m}}}{\partial g^{\mu v} \partial g^{\alpha \beta}} .
$$

For this work, we assume the matter contents as anisotropic fluid distribution defined by the following energy-momentum tensor

$$
\mathcal{T}_{\mu v}=\left(\rho+p_{t}\right) v_{\mu} v_{v}-p_{t} \mathrm{~g}_{\mu v}+\left(p_{r}-p_{t}\right) \xi_{\mu} \xi_{v}
$$

where $v_{\mu}$ and $\xi_{\mu}$ represent the 4 -velocity vectors defined by $v^{\mu}=e^{-\mu} \delta_{0}^{\mu}$ and $\xi^{\mu}=e^{-v} \delta_{1}^{\mu}$ which satisfy the relations $v^{\mu} v_{\mu}=-\xi^{\mu} \xi_{\mu}=1$. Here $\rho, p_{r}$ and $p_{t}$ are the notations for ordinary matter density, radial and tangential pressures, respectively.

The set of field Equation (2) can be further arranged to the following form:

$$
\mathrm{G}_{\mu v}=\frac{1}{f_{\mathscr{R}}(\mathscr{R}, \mathcal{T})}\left(\left(8 \pi+f_{\mathcal{T}}(\mathscr{R}, \mathcal{T})\right) \mathcal{T}_{\mu v}+\left(\nabla_{\mu} \nabla_{v}-g_{\mu \nu} \square\right) f_{\mathscr{R}}(\mathscr{R}, \mathcal{T}) \frac{1}{2}\left(f(\mathscr{R}, \mathcal{T})-\mathscr{R} f_{\mathscr{R}}(\mathscr{R}, \mathcal{T})\right) g_{\mu v}-\rho g_{\mu \nu} f_{\mathcal{T}}(\mathscr{R}, \mathcal{T})\right) .
$$

The geometry of an object exhibiting static and spherically symmetry properties is defined by the line element given by

$$
d s^{2}=-e^{\mu(r)} d t^{2}+e^{v(r)} d r^{2}+r^{2} d \Omega^{2},
$$

where $d \Omega^{2}=d \theta^{2}+\sin ^{2} \theta d \phi^{2}$. Further, we consider an interesting and simple linear model of $f(\mathscr{R}, \mathcal{T})$ modified theory which is defined by 


$$
f(\mathscr{R}, \mathcal{T})=\mathscr{R}+\lambda \mathcal{T}
$$

Utilizing the metric components of Equation (5) in Equation (4), the expressions of energy density function and pressure components take the following form

$$
\begin{aligned}
8 \pi \rho & =\frac{e^{-v(r)}}{4(\lambda+1)(2 \lambda+1) r^{2}}\left(-4 \lambda+4 \lambda r^{2} \mu^{\prime \prime}(r)+(2 \lambda+1) r^{2} \mu^{\prime}(r)^{2}-(\lambda+1)\left(2 r^{2} \mu^{\prime \prime}(r)\right.\right. \\
& \left.-r^{2} \mu^{\prime}(r) v^{\prime}(r)+r^{2} \mu^{\prime}(r)^{2}+4 r \mu^{\prime}(r)-4 r v^{\prime}(r)-4 e^{v(r)}+4\right)+2 r^{2} \mu^{\prime \prime}(r) \\
& \left.-(2 \lambda+1) r \mu^{\prime}(r)\left(r v^{\prime}(r)-4\right)+4 \lambda r v^{\prime}(r)+4 \lambda e^{v(r)}\right) \\
8 \pi p_{r} & =\frac{e^{-v(r)}}{4\left(2 \lambda^{2}+3 \lambda+1\right) r^{2}}\left(-2\left((2 \lambda+1) r^{2} \mu^{\prime \prime}(r)+2 \lambda\left(e^{v(r)}-1\right)\right)+(2 \lambda+1) r^{2} \mu^{\prime}(r) v^{\prime}(r)\right. \\
& -(2 \lambda+1) r^{2} \mu^{\prime}(r)^{2}+(\lambda+1)\left(2 r^{2} \mu^{\prime \prime}(r)-r^{2} \mu^{\prime}(r) v^{\prime}(r)+r^{2} \mu^{\prime}(r)^{2}+4 r \mu^{\prime}(r)\right. \\
& \left.\left.-4 r v^{\prime}(r)-4 e^{v(r)}+4\right)+4(\lambda+1) r v^{\prime}(r)\right), \\
8 \pi p_{t} & =\frac{e^{-v(r)}}{4\left(2 \lambda^{2}+3 \lambda+1\right) r^{2}}\left(( \lambda + 1 ) \left(2 r^{2} \mu^{\prime \prime}(r)-r^{2} \mu^{\prime}(r) v^{\prime}(r)+r^{2} \mu^{\prime}(r)^{2}+4 r \mu^{\prime}(r)-4 r v^{\prime}(r)\right.\right. \\
& \left.\left.-4 e^{v(r)}+4\right)+2\left(-(2 \lambda r+r) \mu^{\prime}(r)+2(\lambda+1)\left(e^{v(r)}-1\right)+r v^{\prime}(r)\right)\right), \\
8 \pi\left(p_{t}-p_{r}\right) & =\frac{e^{-v(r)}}{4(\lambda+1) r^{2}}\left(r\left(2 r \mu^{\prime \prime}(r)+\mu^{\prime}(r)\left(r \mu^{\prime}(r)-r v^{\prime}(r)-2\right)-2 v^{\prime}(r)\right)+4\left(e^{v(r)}-1\right)\right) .
\end{aligned}
$$

Here, the expression $8 \pi\left(p_{t}-p_{r}\right)$ defines the measurement of anisotropy, and is represented by $\triangle$, i.e., $\triangle=8 \pi\left(p_{t}-p_{r}\right)$. The isotopy of the configuration can be achieved in the limit of zero anisotropy factor, i.e., $\triangle=0$.

\subsection{The Karmarker Condition}

Here we shall define the well-known Karmarker condition and the resulting expression of unknown functions. The Karmarkar condition is defined by the relation

$$
\mathscr{R}_{1414}=\frac{\mathscr{R}_{1212} \mathscr{R}_{3434}+\mathscr{R}_{1224} \mathscr{R}_{1334}}{\mathscr{R}_{2323}},
$$

where $\mathscr{R}_{2323} \neq 0$. In this respect, the Pandey-Sharma criterion, i.e., $\mathscr{R}_{2323} \neq 0$ defines a new spacetime with an embedding class-I. Here by correlating the space-time and Karmarker as well as Pande-Sharma condition, we get the following differential equation in terms of metric potentials

$$
v^{\prime}(r) \mu^{\prime}(r)+\mu^{\prime 2}(r)-2\left(\mu^{\prime \prime}(r)+\mu^{\prime 2}(r)\right)=\frac{\mu^{\prime}(r) v^{\prime}(r)}{1-e^{v}},
$$

where we have assumed that $e^{\mu(r)} \neq 1$. Consequently, the solution to the above equation can be written as

$$
e^{\mu(r)}=\left(B \int \sqrt{e^{v(r)}-1} d r+A\right)^{2}
$$

with $B$ and $A$ as arbitrary parameters. Further, we shall find the solution of field Equations (7)-(9) by using the relationship of metric coefficients $g_{t t}$ and $g_{r r}$ given by Equation (13). Let us consider an interesting and significant ansatz for the metric coefficient $g_{r r}$ [53] which is given by

$$
e^{v(r)}=1+\frac{\left(a r^{2}+1\right)^{n} c r^{2}}{\left(b r^{2}+1\right)^{2}},
$$

where $a, b, c$ and $n$ are all arbitrary parameters. Utilizing Equation (14) in Equation (13), we obtain the metric coefficient $g_{t t}$ as follows 


$$
e^{\mu(r)}=\left(\frac{B\left(b r^{2}+1\right)\left(\frac{a b r^{2}+b}{a b r^{2}+a}\right)^{-\frac{n}{2}} \sqrt{\frac{c r^{2}\left(a r^{2}+1\right)^{n}}{\left(b r^{2}+1\right)^{2}}}{ }_{2} F_{1}\left(-\frac{n}{2},-\frac{n}{2} ; 1-\frac{n}{2} ; \frac{a-b}{a b r^{2}+a}\right)}{b n r}+A\right)^{2} .
$$

Further, we substitute the values of $e^{\mu(r)}$ and $e^{\nu(r)}$ from Equations (14) and (15) in the field Equations (7) and (8) and consequently, we get the following expressions for the energy density function, the radial and tangential pressure components and measure of anisotropy parameter:

$$
\begin{aligned}
& 8 \pi \rho=\left(\frac{\left(b r^{2}+1\right)}{\left((\lambda+1)(2 \lambda+1) r\left(a r^{2}+1\right)\left(c r^{2}\left(a r^{2}+1\right)^{n}+\left(b r^{2}+1\right)^{2}\right)^{2}\right.}\right. \\
& \left.\times \frac{\sqrt{\frac{c r^{2}\left(a r^{2}+1\right)^{n}}{\left(b r^{2}+1\right)^{2}}}}{\left(A b n r\left(\frac{a b r^{2}+b}{a b r^{2}+a}\right)^{n / 2}+B\left(b r^{2}+1\right) \sqrt{\frac{c r^{2}\left(a r^{2}+1\right)^{n}}{\left(b r^{2}+1\right)^{2}}} 2 F_{1}\left(-\frac{n}{2},-\frac{n}{2} ; 1-\frac{n}{2} ; \frac{a-b}{a b r^{2}+a}\right)\right)}\right) \\
& \times\left(b n ( b r ^ { 2 } + 1 ) ( \frac { a b r ^ { 2 } + b } { a b r ^ { 2 } + a } ) ^ { n / 2 } \left(A ( 2 \lambda + 1 ) \left(( b r ^ { 2 } + 1 ) \left(r ^ { 2 } \left(a\left(b(2 n-1) r^{2}+2 n+3\right)\right.\right.\right.\right.\right. \\
& \left.-\quad b)+3)+c r^{2}\left(a r^{2}+1\right)^{n+1}\right) \sqrt{\frac{c r^{2}\left(a r^{2}+1\right)^{n}}{\left(b r^{2}+1\right)^{2}}}+B \lambda r\left(( b r ^ { 2 } + 1 ) \left(r ^ { 2 } \left(a \left(b(n+1) r^{2}\right.\right.\right.\right. \\
& \left.\left.+\quad n+3)+b)+3)+2 c r^{2}\left(a r^{2}+1\right)^{n+1}\right)\right)+B c(2 \lambda+1) r\left(a r^{2}+1\right)^{n}\left(( b r ^ { 2 } + 1 ) \left(r^{2}(a(b\right.\right. \\
& \left.\left.\left.\left.\left.\times \quad(2 n-1) r^{2}+2 n+3\right)-b\right)+3\right)+c r^{2}\left(a r^{2}+1\right)^{n+1}\right){ }_{2} F_{1}\left(-\frac{n}{2},-\frac{n}{2} ; 1-\frac{n}{2} ; \frac{a-b}{a b r^{2}+a}\right)\right), \\
& 8 \pi p_{r}=\left(\frac{-\left(b r^{2}+1\right)}{(\lambda+1)(2 \lambda+1) r\left(a r^{2}+1\right)\left(c r^{2}\left(a r^{2}+1\right)^{n}+\left(b r^{2}+1\right)^{2}\right)^{2}}\right. \\
& \left.\times \frac{\sqrt{\frac{c r^{2}\left(a r^{2}+1\right)^{n}}{\left(b r^{2}+1\right)^{2}}}}{\left(A b n r\left(\frac{a b r^{2}+b}{a b r^{2}+a}\right)^{n / 2}+B\left(b r^{2}+1\right) \sqrt{\frac{c r^{2}\left(a r^{2}+1\right)^{n}}{\left(b r^{2}+1\right)^{2}}}{ }_{2} F_{1}\left(-\frac{n}{2},-\frac{n}{2} ; 1-\frac{n}{2} ; \frac{a-b}{a b r^{2}+a}\right)\right)}\right) \\
& \times\left(b n ( b r ^ { 2 } + 1 ) ( \frac { a b r ^ { 2 } + b } { a b r ^ { 2 } + a } ) ^ { n / 2 } \left(A(2 \lambda+1)\left(a r^{2}+1\right)\left(c r^{2}\left(a r^{2}+1\right)^{n}+\left(b r^{2}+1\right)^{2}\right)\right.\right. \\
& \times \sqrt{\frac{c r^{2}\left(a r^{2}+1\right)^{n}}{\left(b r^{2}+1\right)^{2}}}+B r\left(b r^{2}+1\right)\left(r^{2}\left(\lambda\left(a\left(b(n-3) r^{2}+n-1\right)-3 b\right)-2\left(a b r^{2}+a+b\right)\right)\right. \\
& \left.-\quad \lambda-2)-2 B c(\lambda+1) r^{3}\left(a r^{2}+1\right)^{n+1}\right)+B c(2 \lambda+1) r\left(a r^{2}+1\right)^{n+1}\left(c r^{2}\left(a r^{2}+1\right)^{n}\right. \\
& \left.\left.+\left(b r^{2}+1\right)^{2}\right){ }_{2} F_{1}\left(-\frac{n}{2},-\frac{n}{2} ; 1-\frac{n}{2} ; \frac{a-b}{a b r^{2}+a}\right)\right) \text {, }
\end{aligned}
$$




$$
\begin{aligned}
& 8 \pi p_{t}=\left(\frac{c r}{(\lambda+1)(2 \lambda+1) \sqrt{\frac{c r^{2}\left(a r^{2}+1\right)^{n}}{\left(b r^{2}+1\right)^{2}}}\left(c r^{2}\left(a r^{2}+1\right)^{n}+\left(b r^{2}+1\right)^{2}\right)^{2}}\right. \\
& \left.\times \frac{\left(a r^{2}+1\right)^{n-1}}{\left(A b n r\left(\frac{a b b^{2}+b}{a b r^{2}+a}\right)^{n / 2}+B\left(b r^{2}+1\right) \sqrt{\frac{c r^{2}\left(a r^{2}+1\right)^{n}}{\left(b r^{2}+1\right)^{2}}} 2 F_{1}\left(-\frac{n}{2},-\frac{n}{2} ; 1-\frac{n}{2} ; \frac{a-b}{a b r^{2}+a}\right)\right)}\right) \\
& \times \quad\left(b n ( \frac { a b r ^ { 2 } + b } { a b r ^ { 2 } + a } ) ^ { n / 2 } \left(-A(2 \lambda+1)\left(b r^{2}+1\right)\left(a b(n-1) r^{4}+r^{2}(a n+a-b)+1\right) \sqrt{\frac{c r^{2}\left(a r^{2}+1\right)^{n}}{\left(b r^{2}+1\right)^{2}}}\right.\right. \\
& \left.+B r\left(b r^{2}+1\right)\left(r^{2}\left(a\left(\lambda\left(b(n-1) r^{2}+n+1\right)+b n r^{2}+n+2\right)-b \lambda\right)+\lambda+2\right)+B c r^{3}\left(a r^{2}+1\right)^{n+1}\right) \\
& \text { - } \left.B c(2 \lambda+1) r\left(a r^{2}+1\right)^{n}\left(a b(n-1) r^{4}+r^{2}(a n+a-b)+1\right){ }_{2} F_{1}\left(-\frac{n}{2},-\frac{n}{2} ; 1-\frac{n}{2} ; \frac{a-b}{a b r^{2}+a}\right)\right) \text {. } \\
& \Delta=\left(\frac{r\left(b r^{2}+1\right) \sqrt{\frac{c r^{2}\left(a r^{2}+1\right)^{n}}{\left(b r^{2}+1\right)^{2}}}}{8 \pi\left((\lambda+1)\left(a r^{2}+1\right)\left(c r^{2}\left(a r^{2}+1\right)^{n}+\left(b r^{2}+1\right)^{2}\right)^{2}\right)}\right. \\
& \left.\times \frac{\left(c\left(a r^{2}+1\right)^{n+1}-\left(b r^{2}+1\right)\left(b\left(a(n-2) r^{2}-2\right)+a n\right)\right)}{\left(A b n r\left(\frac{a b r^{2}+b}{a b r^{2}+a}\right)^{n / 2}+B\left(b r^{2}+1\right) \sqrt{\frac{c r^{2}\left(a r^{2}+1\right)^{n}}{\left(b r^{2}+1\right)^{2}}}{ }_{2} F_{1}\left(-\frac{n}{2},-\frac{n}{2} ; 1-\frac{n}{2} ; \frac{a-b}{a b r^{2}+a}\right)\right)}\right) \\
& \times\left(b n\left(b r^{2}+1\right)\left(\frac{a b r^{2}+b}{a b r^{2}+a}\right)^{n / 2}\left(A \sqrt{\frac{c r^{2}\left(a r^{2}+1\right)^{n}}{\left(b r^{2}+1\right)^{2}}}-B r\right)+B c r\left(a r^{2}+1\right)^{n}\right. \\
& \left.\times \quad{ }_{2} F_{1}\left(-\frac{n}{2},-\frac{n}{2} ; 1-\frac{n}{2} ; \frac{a-b}{a b r^{2}+a}\right)\right) .
\end{aligned}
$$

\subsection{Boundary Conditions}

In this subsection, we shall define the boundary conditions required by taking the obtained solutions as interior geometry while the exterior geometry is described by the Schwarzschild line-element which is defined as

$$
d s^{2}=-\Psi d t^{2}+\Psi^{-1} d r^{2}+r^{2} d \Omega^{2},
$$

where $\Psi=(1-2 M / r)$ and consequently, $\Psi^{-1}=(1-2 M / r)^{-1}$. In addition, $d \Omega^{2}=d \theta^{2}+\sin ^{2} \theta d \phi^{2}$. Here we shall apply a constraint on the radial coordinate given by $r>r_{s}$, where $r_{s}$ is the Schwarzschild radius. It is worthwhile to specify here that without applying this condition, black hole solutions can be obtained.

Using the metric components $g_{t t}$ and $g_{r r}$ from Equations (14) and (15) over the boundary $r=R$, we obtain the following relations

$$
\begin{aligned}
\left(1-\frac{2 M}{R}\right) & =\left(\frac{B\left(b R^{2}+1\right)\left(\frac{a b R^{2}+b}{a b R^{2}+a}\right)^{-\frac{n}{2}} \sqrt{\frac{c R^{2}\left(a R^{2}+1\right)^{n}}{\left(b R^{2}+1\right)^{2}}}}{b n R}{ }_{2} F_{1}\left(-\frac{n}{2},-\frac{n}{2} ; 1-\frac{n}{2} ; \frac{a-b}{a b R^{2}+a}\right)\right. \\
\left(1-\frac{2 M}{R}\right)^{-1} & =1+\frac{\left(a R^{2}+1\right)^{n} c R^{2}}{\left(a R^{2}+1\right)^{2}}, \\
p_{r}(r=R) & =0 .
\end{aligned}
$$


Further, by taking boundary conditions in Equations (21) and (22) into account, expressions for the unknowns can be obtained as follows

$$
\begin{aligned}
& c=-\frac{2 M\left(b r^{2}+1\right)^{2}\left(a r^{2}+1\right)^{-n}}{r^{2}(2 M-r)}, \\
& A=\frac{\sqrt{r-2 M}}{\sqrt{r}}-\frac{B F\left(\frac{a b r^{2}+b}{a b r^{2}+a}\right)^{-\frac{n}{2}} \sqrt{c\left(a r^{2}+1\right)^{n}}}{b n}, \\
& B=-\frac{b B_{1}(2 \lambda+1) n r \sqrt{r-2 M}}{\sqrt{r}\left(b B_{2} n r\left(b r^{2}+1\right)-B_{3} B_{4} F(2 \lambda+1)\left(a r^{2}+1\right)\right)}
\end{aligned}
$$

where

$$
\begin{aligned}
F & ={ }_{2} F_{1}\left(-\frac{n}{2},-\frac{n}{2} ; 1-\frac{n}{2} ; \frac{a-b}{a b R^{2}+a}\right) \\
B_{1} & =\sqrt{c}\left(a r^{2}+1\right)^{\frac{n}{2}+1}\left(\frac{a b r^{2}+b}{a b r^{2}+a}\right)^{n / 2}\left(c r^{2}\left(a r^{2}+1\right)^{n}+\left(b r^{2}+1\right)^{2}\right), \\
B_{2} & =\left(\frac{a b r^{2}+b}{a b r^{2}+a}\right)^{n / 2}\left(( b r ^ { 2 } + 1 ) \left(r ^ { 2 } \left(\lambda\left(a\left(b(n-3) r^{2}+n-1\right)-3 b\right)\right.\right.\right. \\
& \left.\left.\left.-2\left(a b r^{2}+a+b\right)\right)-\lambda-2\right)-2 c(\lambda+1) r^{2}\left(a r^{2}+1\right)^{n+1}\right), \\
B_{3} & =\left(b r^{2}+1\right) \sqrt{c\left(a r^{2}+1\right)^{n}} \sqrt{\frac{c r^{2}\left(a r^{2}+1\right)^{n}}{\left(b r^{2}+1\right)^{2}}}-c r\left(a r^{2}+1\right)^{n}, \\
B_{4} & =c r^{2}\left(1+a r^{2}\right)^{n}+\left(1+b r^{2}\right)^{2} .
\end{aligned}
$$

Other remaining parameters $a, b$ and $n$ can be considered as free and dimensionless whereas notations $M$ and $R$ refer to the mass and radius of an object, respectively. In this study, for further calculations and discussions, we consider the Vela $X-1\left(1.77 M_{\odot}, 9.56 \mathrm{~km}\right)$ compact star model along with $a=0.001 \mathrm{~km}^{-2}$ and $b=0.0015 \mathrm{~km}^{-2}$.

\section{Graphical Analysis of Some Physical Properties of Presented Model in $f(\mathscr{R}, \mathcal{T})$ Theory}

In the present section, we shall investigate graphically our presented stellar object model in $f(\mathscr{R}, \mathcal{T})$ modified gravity by focusing on some necessary physical properties and conditions satisfied by an astronomical compact star object. For this purpose, we shall concentrate on some physical features of the stellar interior like metric potential, energy density distribution, pressure profile, Zeldovich's condition and gradients of energy density and pressure profile with respect to radial coordinate. In this respect, we shall discuss an astronomical object like Vela $X-1\left(1.77 M_{\odot}, 9.56 \mathrm{~km}\right)$ under the present model. Since the parameter $\lambda$, which is present in the linear form of $f(\mathscr{R}, \mathcal{T})$ function, is very significant as it indicates the deviation from GR, therefore we shall investigate the physical properties of this model by taking two cases of parameter $\lambda$ into account: its small values like $\lambda=0.35$ and large values like $\lambda=2$.

\subsection{Analysis of Some Features of the Model for $\lambda=0.35$}

\subsubsection{Metric Potential}

Herein this segment, we shall describe the graphical behavior of metric potentials for an astronomical object, i.e., $g_{t t}=e^{\mu}$, and $g_{r r}=e^{v}$. From Equations (31) and (33), it can be observed that the metric functions satisfy $e^{\nu(r=0)}=1$ and $e^{\mu(r=0)} \neq 0$, which indicated that our obtained model is physically interesting and acceptable. Further, it can be seen from the left panel of Figure 1 that both metric components exhibit regular, increasing and hence monotonic behavior in the inner side 
of astronomical object. Therefore, it is concluded that the metric potential components satisfy the required necessary condition which ensures physical viability of the obtained model.
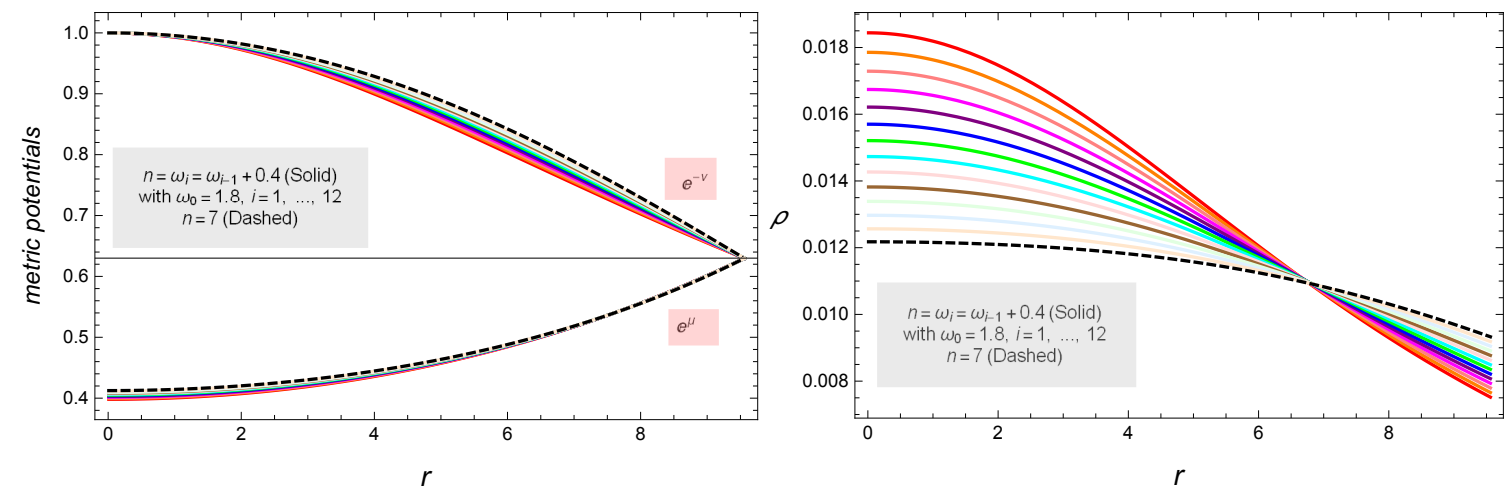

Figure 1. It shows the evolution of metric potentials (left) and energy density function (right) versus $r$ for Vela $X-1$ by using the parameters values from the Table 1 .

\subsubsection{Zeldovich's Condition}

Here, we explore the energy density distribution and radial pressure profile at central values $r=0$, i.e., $\rho(r=0)$ and $p_{r}(r=0)$, which are defined as

$$
\begin{aligned}
\rho_{c} & =\frac{3 c}{8 \pi(\lambda+1)(2 \lambda+1)}, \\
p_{r c} & =\frac{\sqrt{c}\left(b n\left(\frac{b}{a}\right)^{n / 2}(B(\lambda+2)-A \sqrt{c}(2 \lambda+1))-B c(2 \lambda+1){ }_{2} F_{1}\left(-\frac{n}{2},-\frac{n}{2} ; 1-\frac{n}{2} ; 1-\frac{b}{a}\right)\right)}{8 \pi(\lambda+1)(2 \lambda+1)\left(A b n\left(\frac{b}{a}\right)^{n / 2}+B \sqrt{c} F_{2} F_{1}\left(-\frac{n}{2},-\frac{n}{2} ; 1-\frac{n}{2} ; 1-\frac{b}{a}\right)\right)} .
\end{aligned}
$$

The Zeldovich's condition is defined as a ratio of density and pressure given by Equations (27) and (28), i.e., $p_{r c} / \rho_{c}$, which turns out be

$$
\frac{b n\left(\frac{b}{a}\right)^{n / 2}(B(\lambda+2)-A \sqrt{c}(2 \lambda+1))-B c(2 \lambda+1){ }_{2} F_{1}\left(-\frac{n}{2},-\frac{n}{2} ; 1-\frac{n}{2} ; 1-\frac{b}{a}\right)}{3 \sqrt{c}\left(A b n\left(\frac{b}{a}\right)^{n / 2}+B \sqrt{c}{ }_{2} F_{1}\left(-\frac{n}{2},-\frac{n}{2} ; 1-\frac{n}{2} ; 1-\frac{b}{a}\right)\right)} \leq 1 .
$$

It is evident from the Equation (29) and Table 1 that the Zeldovich's condition is satisfied for all suitable values of involved parameters in $f(\mathscr{R}, \mathcal{T})$ gravity under the present model. From the consistency of Zeldovich's condition, it is confirmed that our presented model is physically acceptable for a stellar object.

\subsubsection{Energy Density}

In this segment, we shall explore the graphical behavior of energy density for our obtained model under the Karmarker condition for a compact star Vela $X-1$ in $f(\mathscr{R}, \mathcal{T})$ modified theory. The graphical illustration of energy density has been provided in the right plot of Figure 1 . Here, it is noted that the density function exhibits positive decreasing behavior versus radial coordinate for $r \leq 9.56$ along with $1.8 \leq n<7$ excluding the values $n=2,4$ and $n=6$. In this plot, the solid graphs illustrate the current development of the energy density function for $1.8 \leq n<7$ and the dashed graphs refer to its behavior for $n=7$. 
Table 1. Calculated values of $c, A$ and $B$ under Vela $X-1\left(1.77 M_{\odot}, 9.56 \mathrm{~km}\right)$ with $a=0.001 \mathrm{~km}^{-2}, b=0.0015 \mathrm{~km}^{-2}$ and $\lambda=0.35$ for different suitable values of $n$

\begin{tabular}{ccccc}
\hline $\mathbf{n}$ & $\mathbf{c}$ & $\mathbf{A}$ & $\mathbf{B}$ & $p_{r c} / \rho_{c}$ \\
\hline 1.8 & 0.00711 & 3.48530 & 0.04329 & 0.48013 \\
\hline 2.2 & 0.00686 & -4.27640 & 0.04342 & 0.50382 \\
\hline 2.6 & 0.00663 & -1.36860 & 0.04355 & 0.52824 \\
\hline 3.0 & 0.00639 & -0.62393 & 0.04368 & 0.55319 \\
\hline 3.4 & 0.00618 & -0.12125 & 0.04381 & 0.55319 \\
\hline 3.8 & 0.00596 & 0.92485 & 0.04394 & 0.60458 \\
\hline 4.2 & 0.00576 & -1.41630 & 0.04407 & 0.63089 \\
\hline 4.6 & 0.00556 & -0.46114 & 0.04421 & 0.65790 \\
\hline 5.0 & 0.00537 & -0.18517 & 0.04433 & 0.68546 \\
\hline 5.4 & 0.00518 & 0.00922 & 0.04447 & 0.71351 \\
\hline 5.8 & 0.00500 & 0.36662 & 0.04466 & 0.74225 \\
\hline 6.2 & 0.00483 & -0.33038 & 0.04474 & 0.77137 \\
\hline 6.6 & 0.00467 & -0.00888 & 0.04487 & 0.80142 \\
\hline 7.0 & 0.00451 & 0.09896 & 0.04502 & 0.83202 \\
\hline
\end{tabular}

\subsubsection{Pressure}

Here, we shall provide the graphical behavior of radial and tangential pressure profiles for a Vela $X-1$ compact star model representing a new family of solutions under the Karmarker condition in $f(\mathscr{R}, \mathcal{T})$ theory. The graphical development of radial and pressure components have been presented in Figure 2. It can be seen from these plots of Figure 2 that both radial and tangential pressures admit positive decreasing behavior for the radial coordinate satisfying $r \leq 9.56$ with $1.8 \leq n<7$ (excluding even numbers between $1.8 \leq n<7$ ).
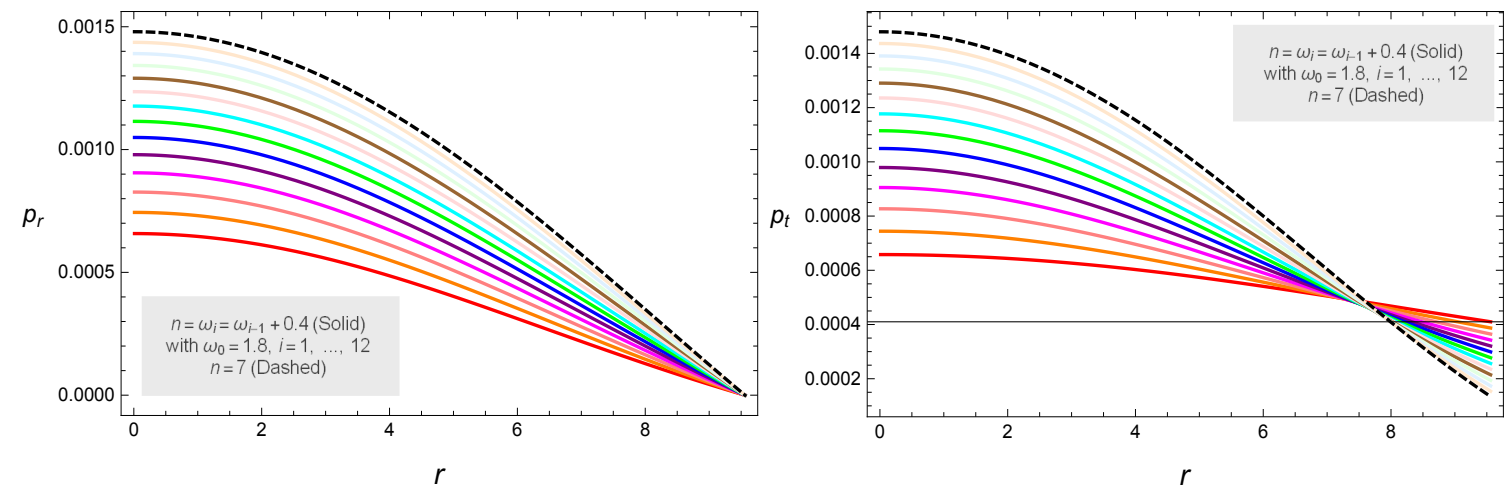

Figure 2. Provides the graphical response of radial (left) and tangential pressures (right) versus $r$ for Vela $X-1$, using values of parameters From the Table 1 .

\subsubsection{Anisotropy Distribution}

In this part, we shall describe the graphical behavior of anisotropy measurement parameter denoted by $\triangle$ versus radial coordinate which is provided in the left panel of Figure 3 . It indicates that the measure of anisotropy distribution, i.e., $\triangle=8 \pi\left(p_{t}-p_{r}\right)$ is positive throughout the region of the compact star Vela $X-1$ in $f(\mathscr{R}, \mathcal{T})$ modified theory of gravity. It is observed that for $\triangle>0$, i.e., $p_{t}>p_{r}$, the anisotropic force is seen as outward-directed while for $\triangle<0$, this force is inward-directed. Since, in the present study, as we have $\triangle>0$ and consequently, it indicates that the anisotropic pressure is repulsive and hence supports to the structure of compact object. 

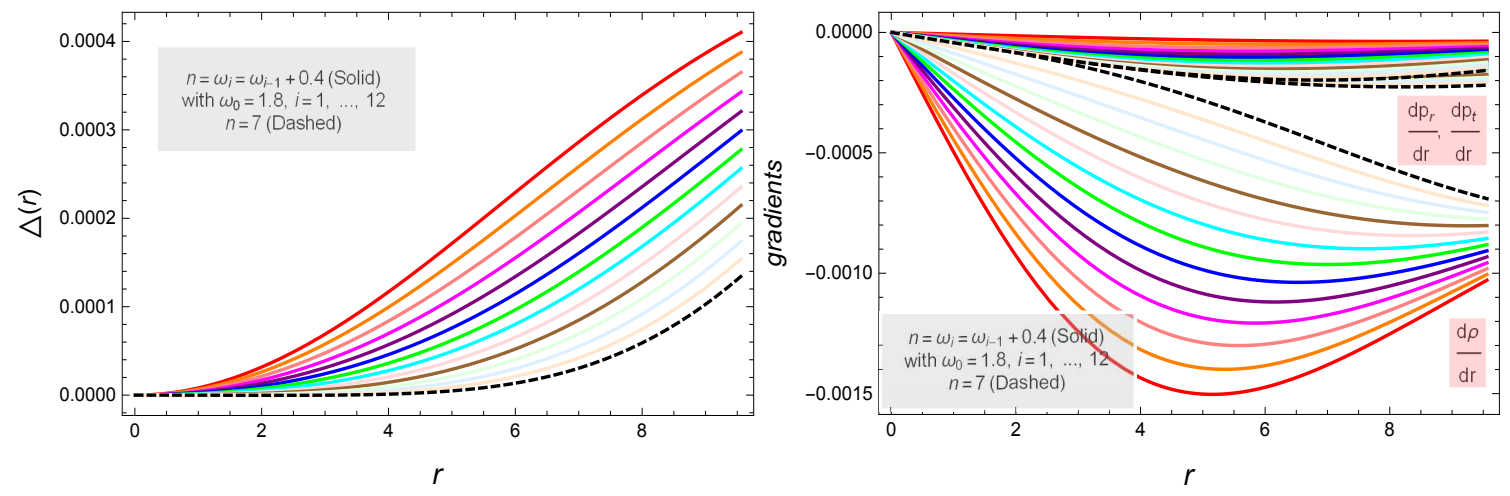

Figure 3. Describes the graphical behavior of anisotropy function (left) and radial derivatives of $\rho$ and $p_{r}$ (right) versus $r$ for Vela $X-1$, by using the parameters values from the Table 1 .

\subsubsection{Gradients}

Here, we shall present the graphical illustration of radial derivatives of energy density and radial pressure profile, i.e., $d \rho / d r, d p_{r} / d r$. The corresponding graphical illustration is provided in the right panel of Figure 3. Here seperate visible curves refer to the derivatives of the energy density function, while the overlapping curves correspond to the derivative of pressure profiles with respect to radial coordinate for a Vela X - 1 compact object. From this graph, it can be easily observed that all curves representing derivatives show negative decreasing response and consequently, satisfy the primary conditions, i.e., $d \rho / d r<0, d p_{r} / d r<0$ and $d p_{t} / d r<0$. It is also seen that these derivatives are zero at the central radius, i.e., $d \rho(r=0) / d r=0, d p_{r}(r=0) / d r=0$, and $d p_{t}(r=0) / d r=0$. Hence it can be concluded that these gradients meet the required constraints in this modified theory.

\subsubsection{Equilibrium Condition}

In this section, we shall explore the stability of the obtained new family of solutions by taking the equilibrium equation into account. For this purpose, we shall employ a well-known and fundamental equation, known as Tolman-Oppenheimer-Volkov (TOV) equation for checking the stability of the present configuration. This equation is given as follows

$$
\frac{2}{r}\left(p_{t}-p_{r}\right)-\frac{d p_{r}}{d r}-\frac{\mu^{\prime}(r)}{2}\left(\rho+p_{r}\right)=0,
$$

The above equation describes the equilibrium condition by considering three forces namely anisotropic, hydrostatic and gravitational forces into account. The forces are defined by the following mathematical relations:

$$
\mathscr{F}_{\mathrm{a}}=\frac{2}{r}\left(p_{t}-p_{r}\right), \quad \mathscr{F}_{\mathrm{h}}=-\frac{d p_{r}}{d r}, \quad \mathscr{F}_{g}=-\frac{\mu^{\prime}(r)\left(\rho+p_{r}\right)}{2}
$$

and consequently, Equation (30) takes the form

$$
\mathscr{F}_{\mathrm{a}}+\mathscr{F}_{\mathrm{h}}+\mathscr{F}_{\mathrm{g}}=0 .
$$

Further, we discuss the graphical response of these three compulsory forces in reference to the equilibrium equation. In this respect, the behavior of anisotropic force is provided in the left panel of Figure 4, while the plot of gravitational force is given in its right panel. Likewise, the graphical response of hydrostatic force is presented in the left panel of Figure 5, while the stability of the configuration can be observed by the graphical representation of equilibrium condition provided in the right panel of Figure 5. It can be easily observed that these forces balance each other's effect for Vela X -1 in $f(\mathscr{R}, \mathcal{T})$ modified theory of gravity and hence the stability of the model has been achieved under the 
mass and radii which are described in Table 1 . Thus, another necessary required condition is satisfied in our case.
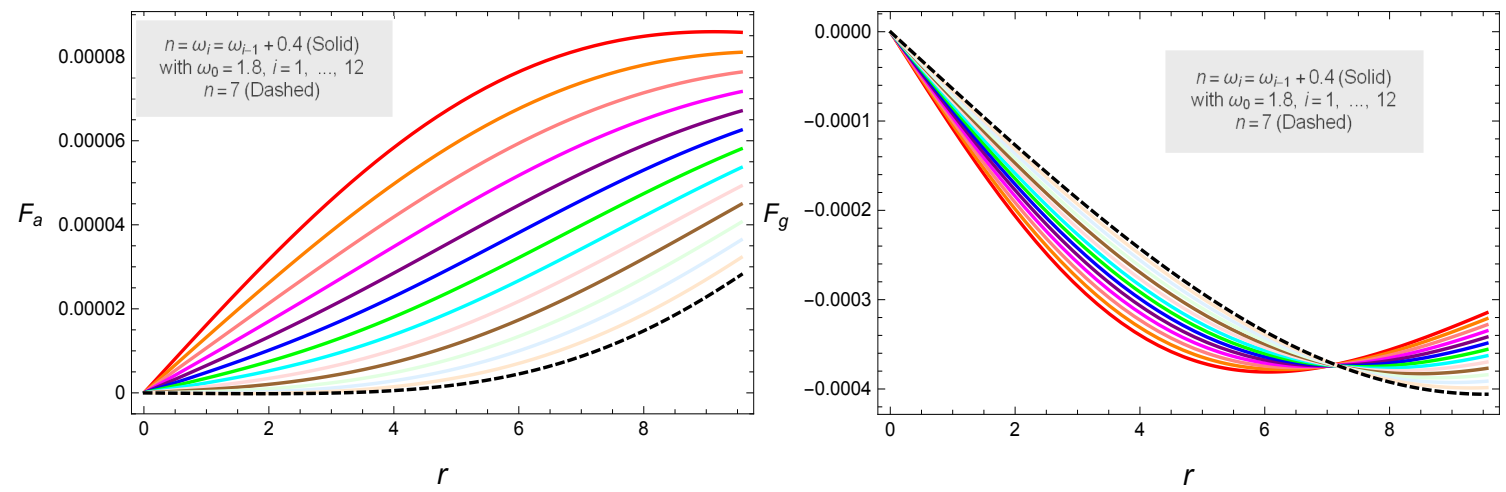

Figure 4. Represents the behavior of forces $\mathscr{F}_{\mathrm{a}}$ (left) and $\mathscr{F}_{g}$ (right) versus $r$ for Vela $X-1$ by using the parameters values from the Table 1.
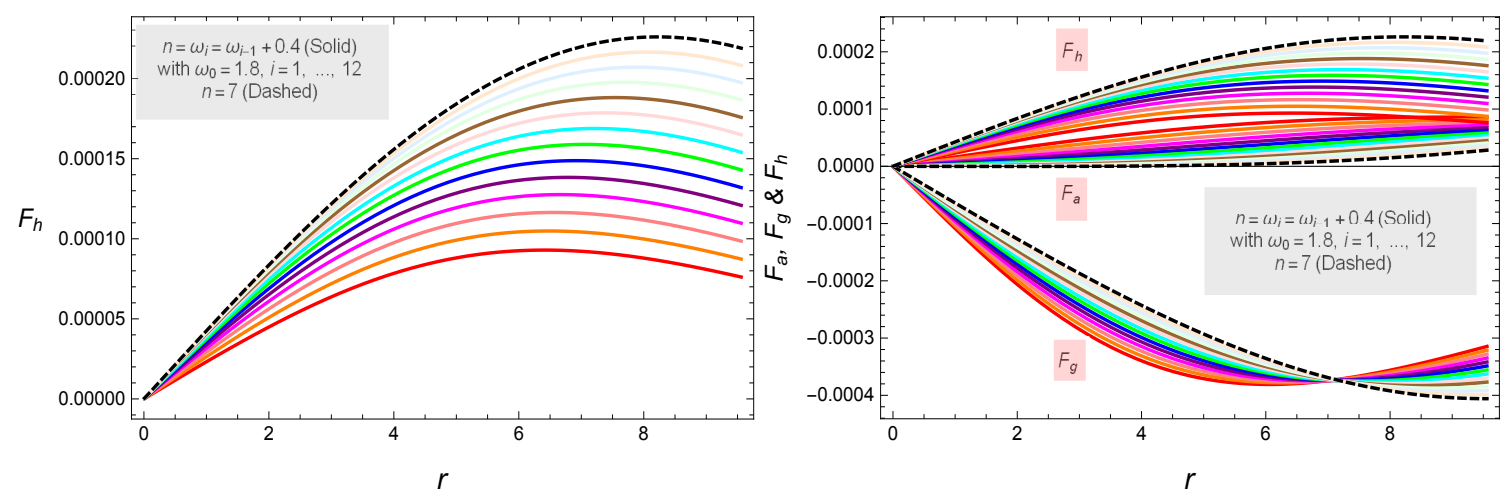

Figure 5. Illustrates the behavior of $\mathscr{F}_{\mathrm{h}}$ (left) and the balanced combine effect of $\mathscr{F}_{\mathrm{a}}, \mathscr{F}_{\mathrm{h}}$ and $\mathscr{F}_{\mathrm{g}}$ (right) versus $r$ for Vela $X-1$ by using the parameters values from the Table 1.

\subsubsection{Energy Conditions}

The study of energy constraints for checking the viability of a constructed model in GR as well as modified theories is regarded as one of the most interesting topic. These constraints also help to fix the values of arbitrary constants. These constraints are defined in terms four different inequalities which are labeled as DEC, SEC, WEC and NEC and are defined as

$$
\begin{aligned}
\text { NEC }: & \rho \geq 0, & \text { WEC }: & \rho-p_{t} \geq 0, \quad \rho-p_{r} \geq 0, \\
\text { SEC }: & \rho-p_{r}-2 p_{t} \geq 0, & D E C: & \rho>\left|p_{r}\right|, \quad \rho>\left|p_{t}\right| .
\end{aligned}
$$

Here, we discuss the graphical response of these different energy conditions in the connection of NEC, WEC, SEC and DEC. The NEC and DEC expressions have been already discussed in the previous section of energy density and pressure profile which indicated that these constraints are consistent in our case. Further, the graphical illustration of remaining WEC and SEC constraints are provided in the right and left panels of Figures 6 and 7. It can be easily observed that these all energy bounds remain satisfied in the current scenario which is a necessary condition for the physical acceptance of compact stars under the observational data (as given in Table 1) in $f(\mathscr{R}, \mathcal{T})$ theory. 

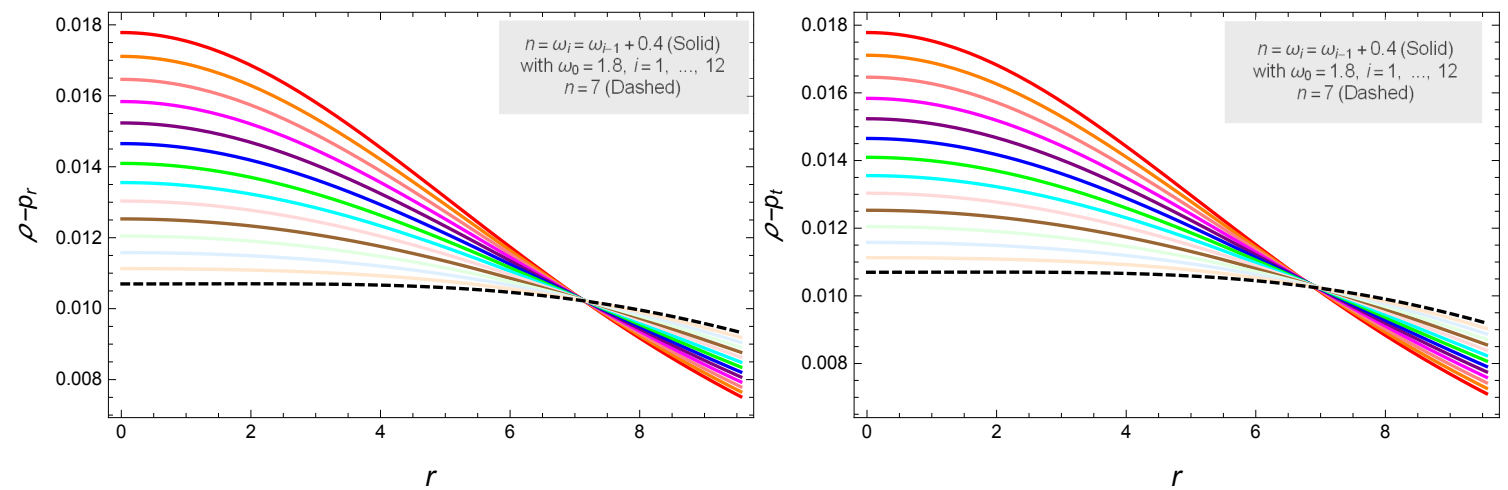

Figure 6. Shows the evolution of $\rho-p_{r}$ (left) and $\rho-p_{t}$ (right) versus $r$ by using the parameters values from the Table 1.
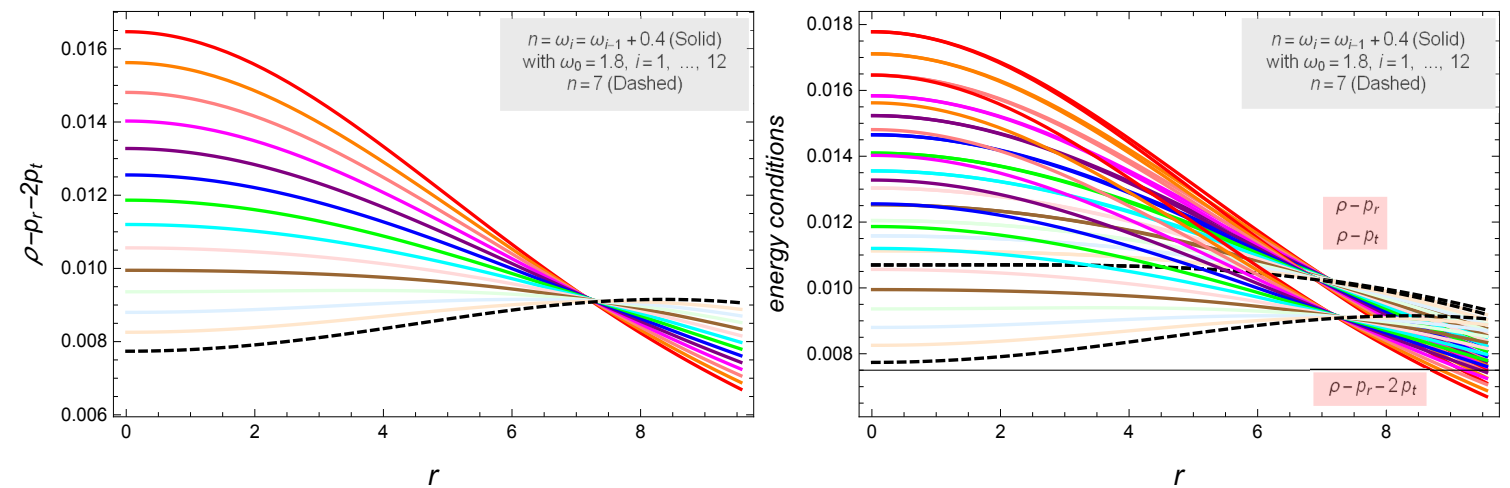

Figure 7. Corresponds to the behavior of $\rho-p_{r}-2 p_{t}$ (left) and all energy constraints together (right) against radial coordinate for Vela $\mathrm{X}-1$.

\subsubsection{Mass Function, Compactness Parameter and Surface Red-Shift Function}

In this segment, we shall discuss the relation of mass and radius of a compact star graphically. In this connection, we shall discuss mass function, mass-radius ratio and surface red-shift function, which are listed below in detail.

The expression of mass function for a compact star with radius $r$ is defined as

$$
m(r)=4 \pi \int_{0}^{r}\left(r^{2} \rho\right) d r
$$

In the current scenario, we use the radius under our presented model and the corresponding graph is presented in the left part of Figure 8. It can be easily observed that the mass function exhibits positive increasing behavior throughout the interior of stellar object, i.e., $m(r)>0$, for $r \leq 9.56$. Further, it can be noticed that $m(r) \rightarrow 0$ as $r \rightarrow 0$ and consequently, it can be concluded that this condition leads to the regularity of the mass function at the center.

Next we shall discuss graphically the mass-radius function, i.e., compactness parameter, which is defined by the following mathematical expression

$$
u(r)=\frac{2 m(r)}{r} .
$$

From the right part of Figure 8, the graphical development of compactness parameter can be noticed which indicates that parameter $u(r)$ remains positive and increasing. It is also easy to verify from this graph that the mass-radius function satisfy the Bhuchdahl [54] condition, i.e., $u(r)<8 / 9$.

Now, with the help of mass-radius function, we investigate the graphical response of surface red-shift function for the considered stellar object, which is defined as 


$$
Z_{s}=\frac{1}{\sqrt{1-2 u(r)}}-1
$$

The graphical response of surface red-shift function, i.e., $Z_{s}$ against the radial coordinate $r$ is presented in the left panel of Figure 9. From this graph, it is found that $Z_{s}$ vanishes at $r=0$ and gradually increases with the increasing values of $r$. It is also worthwhile to mention here that in our case, $Z_{s}$ satisfy the Bohmer and Harko [55] condition under the anisotropic configuration, i.e., $Z_{s}<5$.
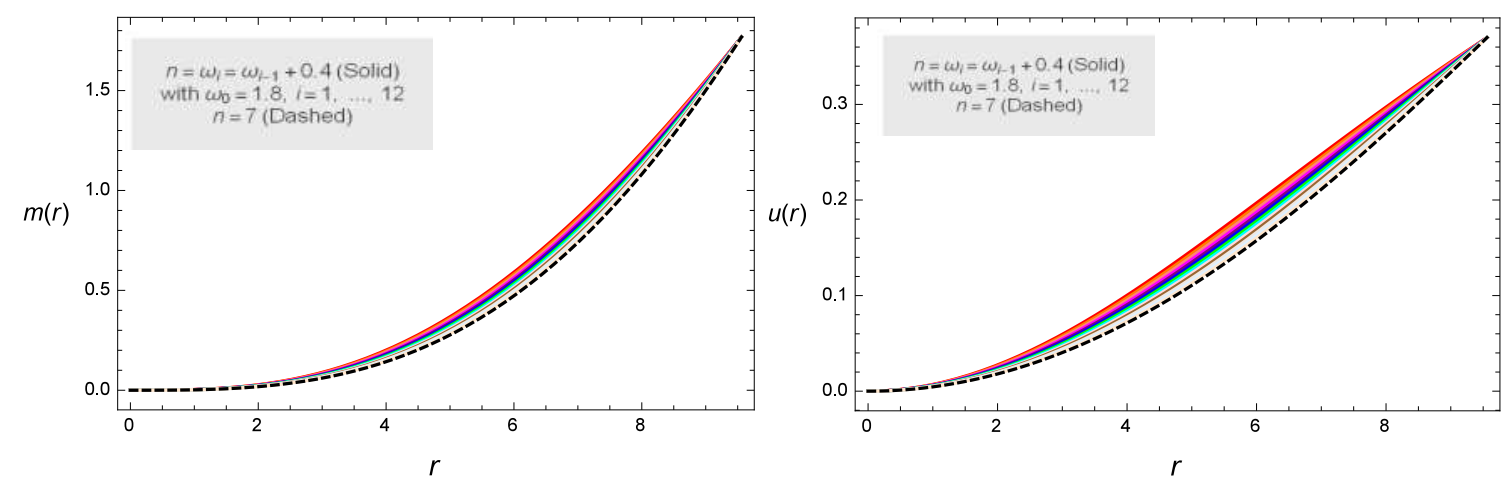

Figure 8. Shows the graphical behavior of mass-radius relation function (left) and compactness parameter (right) versus $r$ for Vela $X-1$.
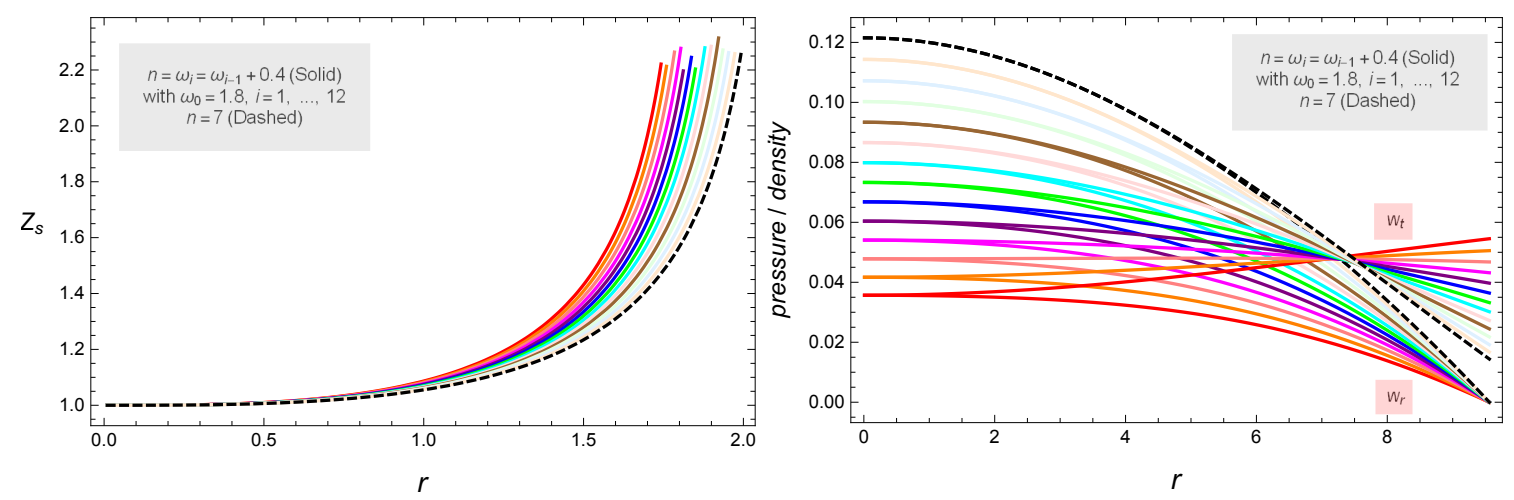

Figure 9. Provides the graph of surface red-shift function (left) and equations of state (right) versus $r$ for Vela X-1.

\subsubsection{Equation of State}

In this segment, we calculate the equations of state for both radial and tangential components of pressure, which are denoted by $w_{r}$ and $w_{t}$, respectively. These parameters are defined in the normal and transverse directions as follows

$$
\begin{aligned}
& w_{r}=\frac{p_{r}}{\rho} \\
& w_{t}=\frac{p_{t}}{\rho} .
\end{aligned}
$$

These two ratios define the equations of state. Here, we explore the graphical response of these two ratios, i.e., $w_{r}$ and $w_{t}$, in the reference of the equation of state parameter. It is noticed from the right part of Figure 9, that both these ratios are less than 1, which is a mandatory condition for a stellar object. It is also shown that both ratios exhibit decreasing behaviors under the current scenario. Hence it can be concluded that the equations of state satisfy the necessary requirement in $f(\mathscr{R}, \mathcal{T})$ theory for Vela $X-1$ model. 


\subsubsection{Causality Stability Analysis}

In this segment, we shall describe the causality stability analysis for the obtained solutions. In this reference, we shall discuss two sorts of the speed of sound parameter (radial and tangential) for Vela $X-1$ compact star model. These speed of sound parameters are represented by $v_{t}^{2}$ and $v_{r}^{2}$ for radial and tangential components, respectively and can be calculated as

$$
v_{t}^{2}=\frac{d p_{t}}{d \rho}, \quad v_{r}^{2}=\frac{d p_{r}}{d \rho} .
$$

It is worthwhile to mention here that the casuality stability of a configuration can be achieved if both the tangential and radial speed of sound parameters take values less than 1 . In addition, the difference between these velocities denoted by $v_{t}^{2}-v_{r}^{2}$ must satisfy Abrea condition [56,57], which is given by $-1 \leq v_{t}^{2}-v_{r}^{2} \leq 0$.

For the present case, the graphs of these parameters are provided in Figures 10 and 11. It is observed form the left part of Figure 10 that the expression $v_{r}^{2}$ attains values less than 1 . It is also noted from the right part of Figure 10 that the expression $v_{t}^{2}$ takes values less than 1 . Further, it is seen that for $1.8 \leq n<7$, both the radial and tangential velocities satisfy the necessary condition, but for $n=7$, both radial and tangential velocities take values greater than 1 and hence violate the necessary condition in this case. Furthermore, it can be noticed from Figure 10 that our model is not compatible with the causality stability criterion for $n=7$, which is represented by dashed graphs for both velocities. From the left panel of Figure 11, it can be observed that the difference $v_{t}^{2}-v_{r}^{2}$ is consistent with the condition $-1 \leq v_{t}^{2}-v_{r}^{2} \leq 0$. Hence it can be concluded that our obtained model is potentially stable.
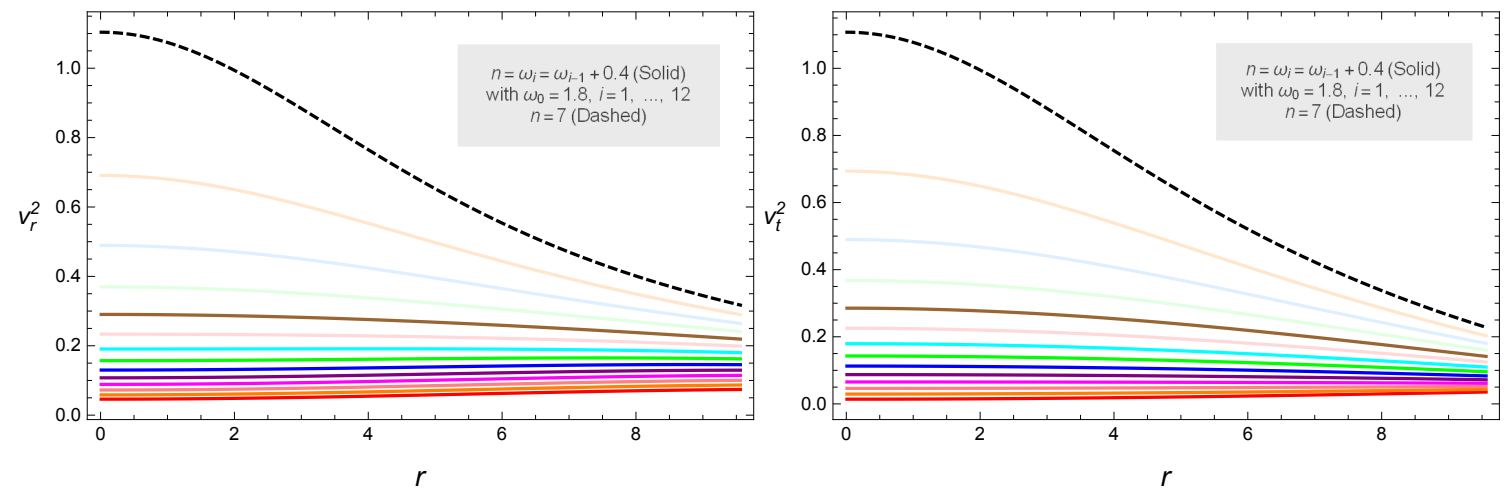

Figure 10. Represents the behavior of $v_{r}^{2}$ (left) and $v_{t}^{2}$ (right) against $r$ for Vela $X-1$.
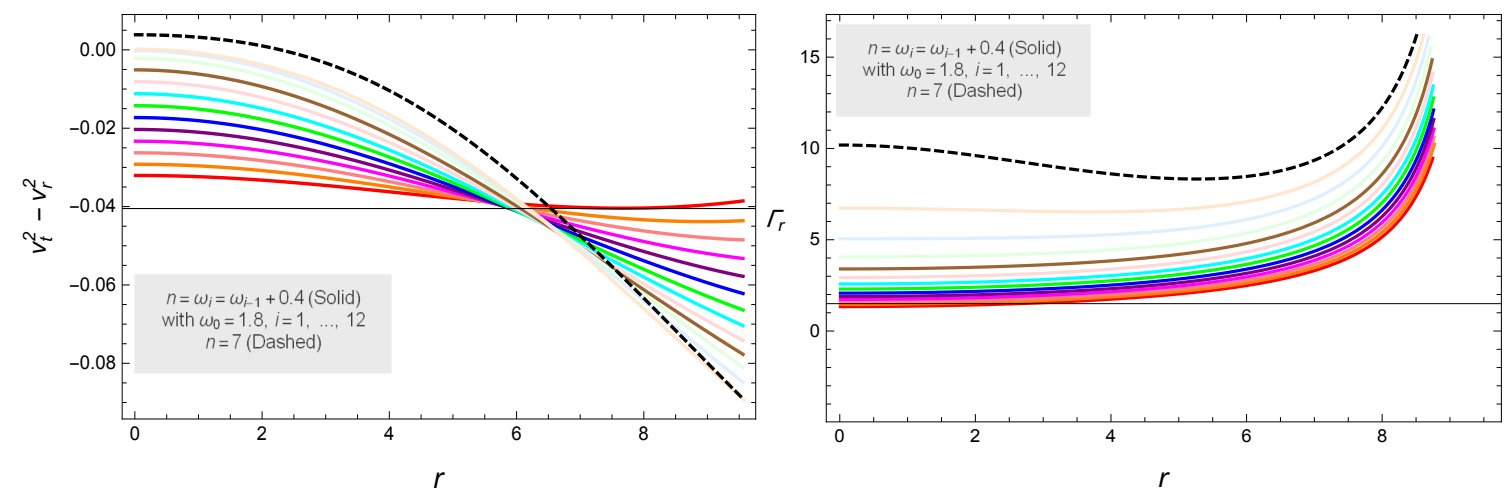

Figure 11. Shows the plots of $v_{t}^{2}-v_{r}^{2}$ (left) and $\Gamma_{r}$ (right) versus $r$ by using the parameters values from the Table 1 . 
3.1.12. The Adiabatic Index Stability Analysis

In this portion, we shall explore an important and necessary ratio of two specific heats given by

$$
\Gamma_{r}=v_{r}^{2} \frac{\rho+p_{r}}{p_{r}} .
$$

It is significant to mention here that the stability of Newtonian sphere can be obtained under the restriction of $\Gamma_{r}>4 / 3$. In literature, it is pointed out that this expression is used to define a neutral equilibrium when the condition $\Gamma_{r}=4 / 3$ holds. In the present case, the graphical development of $\Gamma_{r}$ parameter is provide in the right panel of Figure 11. It is easy to check that the relation $\Gamma_{r}$ shows monotonic increasing behavior for $n<7$ and also is compatible with the condition $\Gamma_{r}>4 / 3$ for Vela $X-1$ compact star, while it exhibits decreasing trend for $n=7$. Hence, the adiabatic index is satisfied with the stability condition in the reference of $f(\mathscr{R}, \mathcal{T})$ theory for $n<7$. In Table 2, we have presented a summary of our obtained solutions. All the physical properties can be seen from Table 2 .

Table 2. Calculated results for Vela $X-1$ compact star under Karmarkar condition in $f(\mathscr{R}, \mathcal{T})$ theory for $\lambda=0.35$.

\begin{tabular}{|c|c|c|}
\hline Expressions & $1.8 \leq n<7$ & $n=7$ \\
\hline$\rho$ & $\rho>0$, satisfied & $\rho>0$, satisfied \\
\hline$p_{r}$ & $p_{r}>0$, satisfied & $p_{r}>0$, satisfied \\
\hline$p_{t}$ & $p_{t}>0$, satisfied & $p_{t}>0$, satisfied \\
\hline$p_{r c} / \rho_{c}$ & $p_{r c} / \rho_{c} \leq 1$ & $p_{r c} / \rho_{c} \leq 1$ \\
\hline$\triangle(r)$ & $\triangle(r)>0$, satisfied & $\triangle(r)>0$, satisfied \\
\hline$d \rho / d r$ & $d \rho / d r<0$, satisfied & $d \rho / d r<0$, satisfied \\
\hline$d p_{r} / d r$ & $d p_{r} / d r<0$, satisfied & $d p_{r} / d r<0$, satisfied \\
\hline $\mathscr{F}_{\mathrm{a}}, \mathscr{F}_{\mathrm{h}}$ and $\mathscr{F}_{\mathrm{g}}$ & forces are Balance & forces are Balance \\
\hline$\rho-p_{r}$ & $\rho-p_{r}>0$, satisfied & $\rho-p_{r}>0$, satisfied \\
\hline$\rho-p_{t}$ & $\rho-p_{t}>0$, satisfied & $\rho-p_{t}>0$, satisfied \\
\hline$\rho-p_{r}-2 p_{t}$ & $\rho-p_{r}-2 p_{t}>0$, satisfied & $\rho-p_{r}-2 p_{t}>0$, satisfied \\
\hline$m(r)$ & $m(r)>0$, satisfied & $m(r)>0$, satisfied \\
\hline$u(r)$ & $0<u(r)<8 / 9$ satisfied & $0<u(r)<8 / 9$ satisfied \\
\hline$Z_{s}$ & $0<Z_{s}<5$, satisfied & $0<Z_{s}<5$, satisfied \\
\hline$w_{r}$ & $0<w_{r}<1$, satisfied & $0<w_{r}<1$, satisfied \\
\hline$w_{t}$ & $0<w_{t}<1$, satisfied & $0<w_{t}<1$, satisfied \\
\hline$v_{r}^{2}$ & $v_{r}^{2}<1$, satisfied & $v_{r}^{2}>1$, not-satisfied \\
\hline$v_{t}^{2}$ & $v_{t}^{2}<1$, satisfied & $v_{t}^{2}>1$, not-satisfied \\
\hline$v_{t}^{2}-v_{r}^{2}$ & $-1 \leq v_{t}^{2}-v_{r}^{2} \leq 0$, satisfied & $-1 \leq v_{t}^{2}-v_{r}^{2} \leq 0$, satisfied \\
\hline$\Gamma_{r}$ & $0<\Gamma_{r}<4 / 3$, satisfied & $0<\Gamma_{r}<4 / 3$, satisfied \\
\hline
\end{tabular}

\subsection{Graphical Analysis of Some Physical Properties for $\lambda=2$}

Here we shall investigate the behavior of presented model by exploring different physical properties graphically (as discussed in previous subsections) for large values of parameter $\lambda$. For this purpose, we fix the parameter as $\lambda=2$ and the corresponding calculated values of unknowns $c, A$ and $B$ under Vela $X-1\left(1.77 M_{\odot}, 9.56 \mathrm{~km}\right)$ are provided in Table 3. Further the graphical analysis of different properties of our obtained solution is given in Figures 12-22. From Figure 12, it is easy to verify that the metric potentials satisfy the necessary condition required for a realistic compact star 
model. Further, the density function shows positive decreasing behavior versus radial coordinate which is also physically acceptable. In Figure 13, it can be seen that radial pressure exhibits physically valid behavior while the tangential pressure shows negative behavior near the boundary and hence refers to non-realistic compact star model. The measure of anisotropy parameter takes negative values as shown in Figure 14 which corresponds to $p_{t}<p_{r}$ and hence the force on the compact star will be inward directed. In addition, the radial derivatives of density and radial pressure exhibit negative decreasing behavior which also favor to a realistic model. The graphical response of gravitational, hydrostatic and anisotropic forces is provided in Figures 15 and 16 which indicate the stability of compact star configuration.
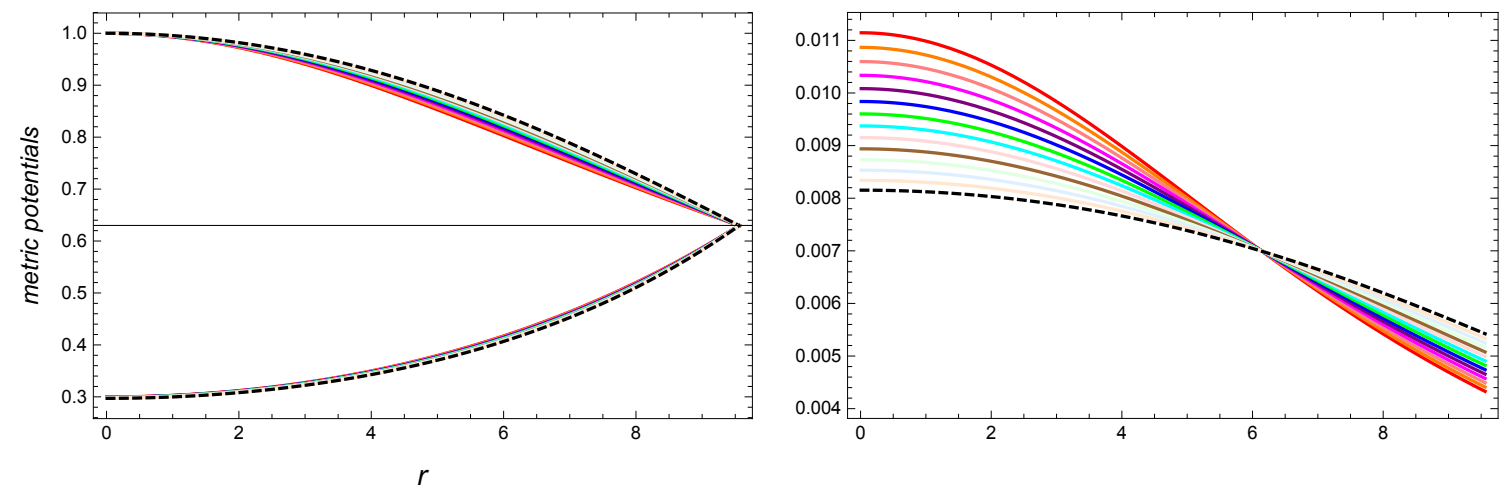

Figure 12. Shows the evolution of metric potential (left) and energy density function (right) against $r$ for Vela $X-1$, by using the parameters values from the Table 3.
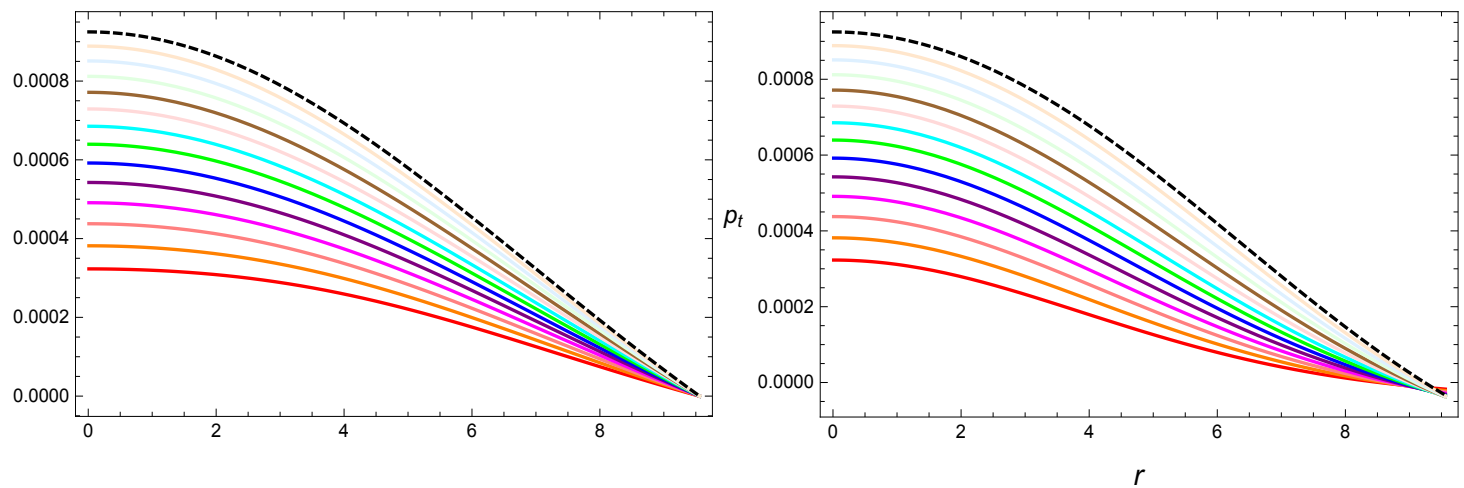

Figure 13. Provides the behavior of radial pressure (left) and tangential pressure (right) versus $r$ for Vela $X-1$ by using the parameters values from the Table 3.
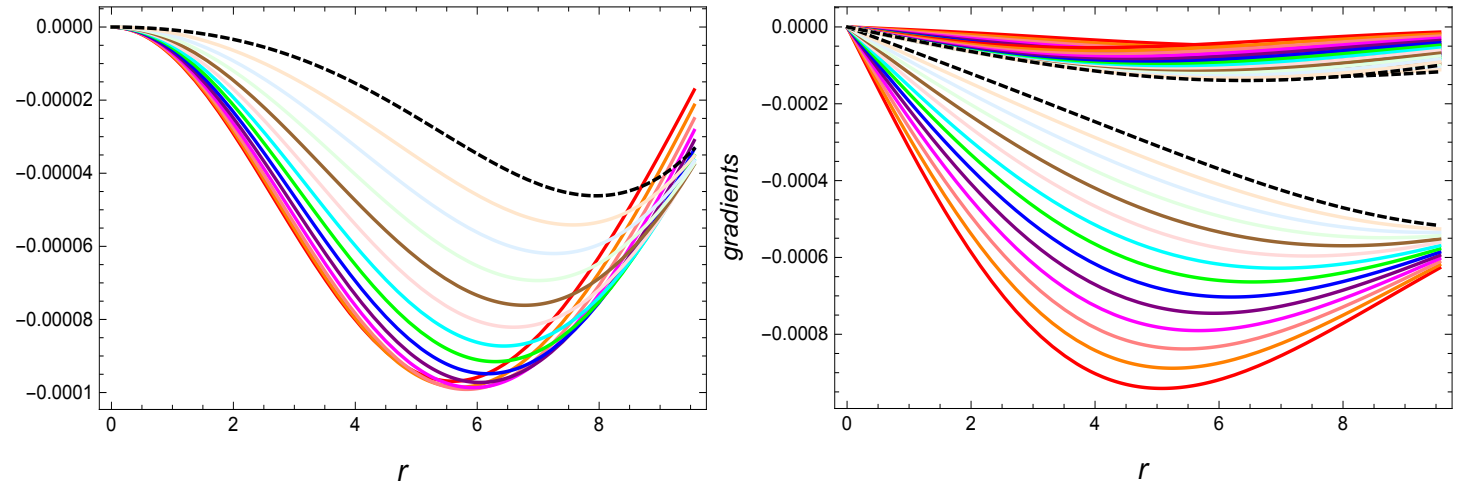

Figure 14. Describes the graphical response of anisotropy function (left) and radial derivative of $\rho$ and $p_{r}$ (right) versus $r$ for Vela $X-1$, by using the parameters values from the Table 3. 

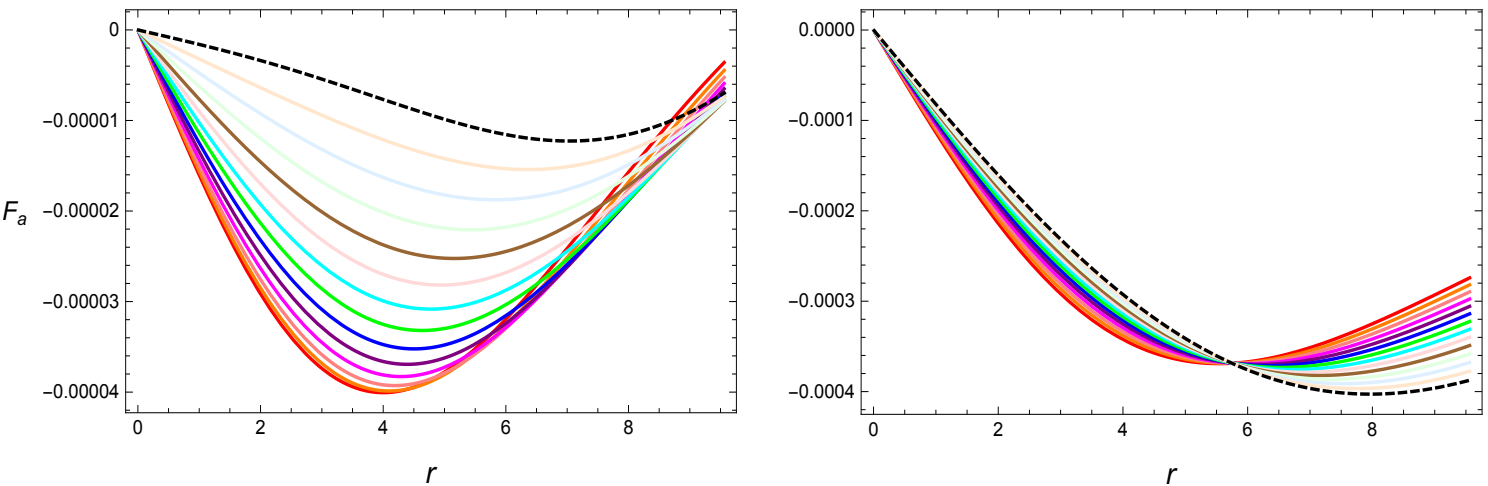

Figure 15. Refers to the plots of forces $\mathscr{F}_{\mathrm{a}}$ (left) and $\mathscr{F}_{g}$ (right) versus $r$ for Vela $X-1$, by using the parameters values from the Table 3 .
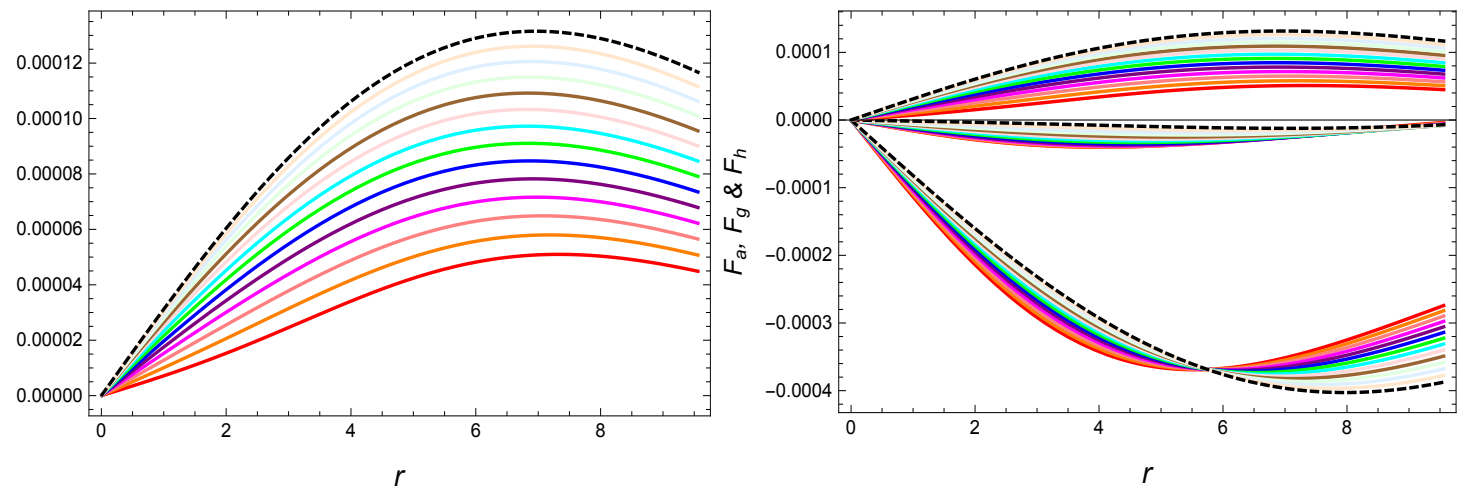

Figure 16. the behavior of $\mathscr{F}_{\mathrm{h}}$ (left) and the combine effect of $\mathscr{F}_{\mathrm{a}}, \mathscr{F}_{\mathrm{h}}$ and $\mathscr{F}_{\mathrm{g}}$ (right) versus $r$ by using the parameters values from the Table 3.
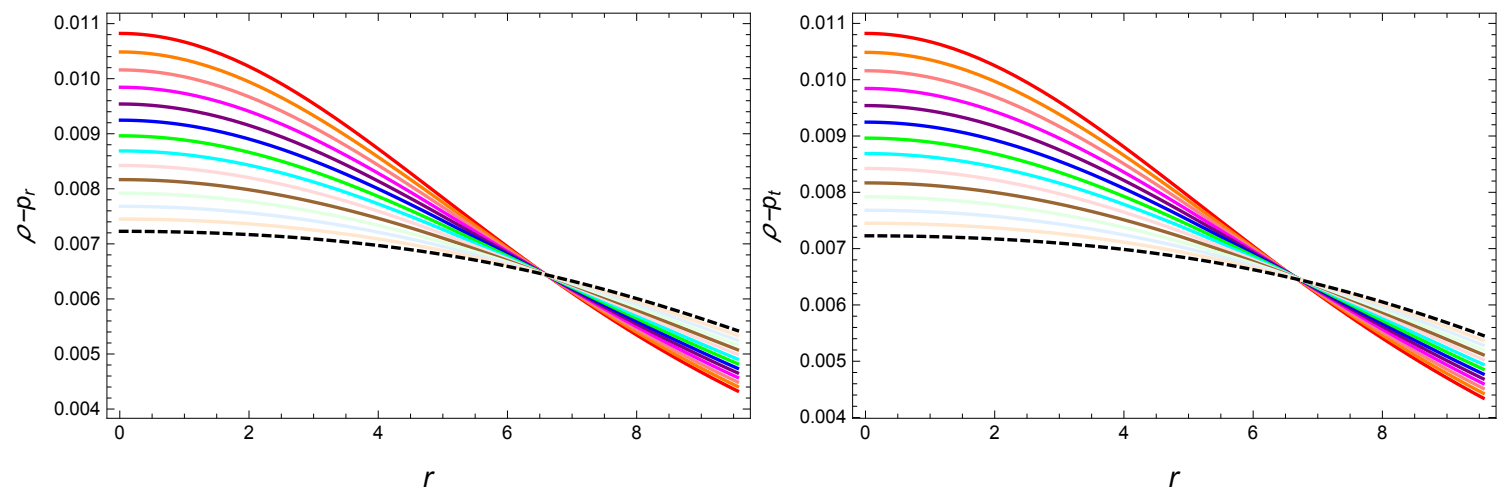

Figure 17. Shows the evolution of $\rho-p_{r}$ (left) and $\rho-p_{t}$ (right) against $r$.
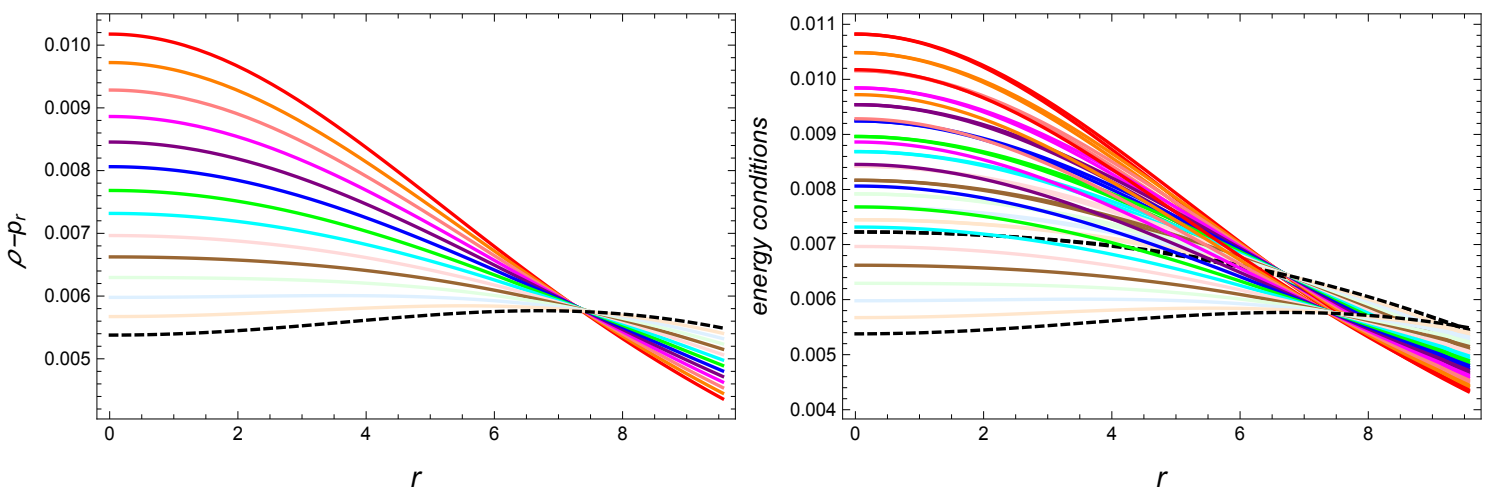

Figure 18. Describes the graphs of $\rho-p_{r}-2 p_{t}$ (left) and all energy conditions (right) against $r$. 

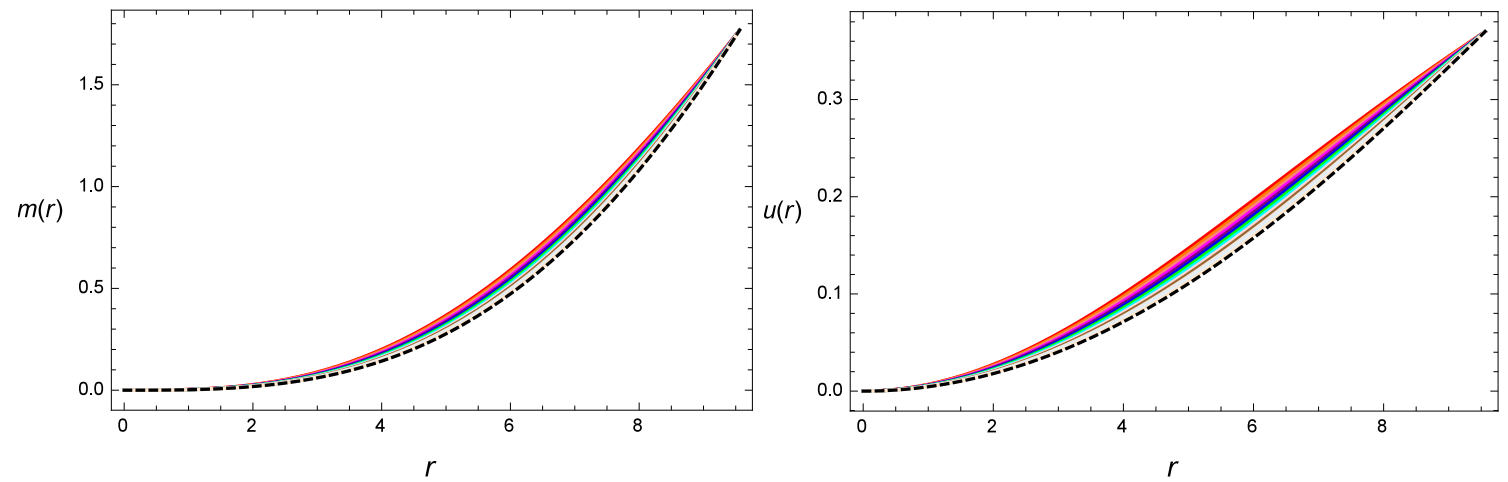

Figure 19. Shows the graphical behavior of mass-radius relation (left) and compactness parameter (right) versus $r$.
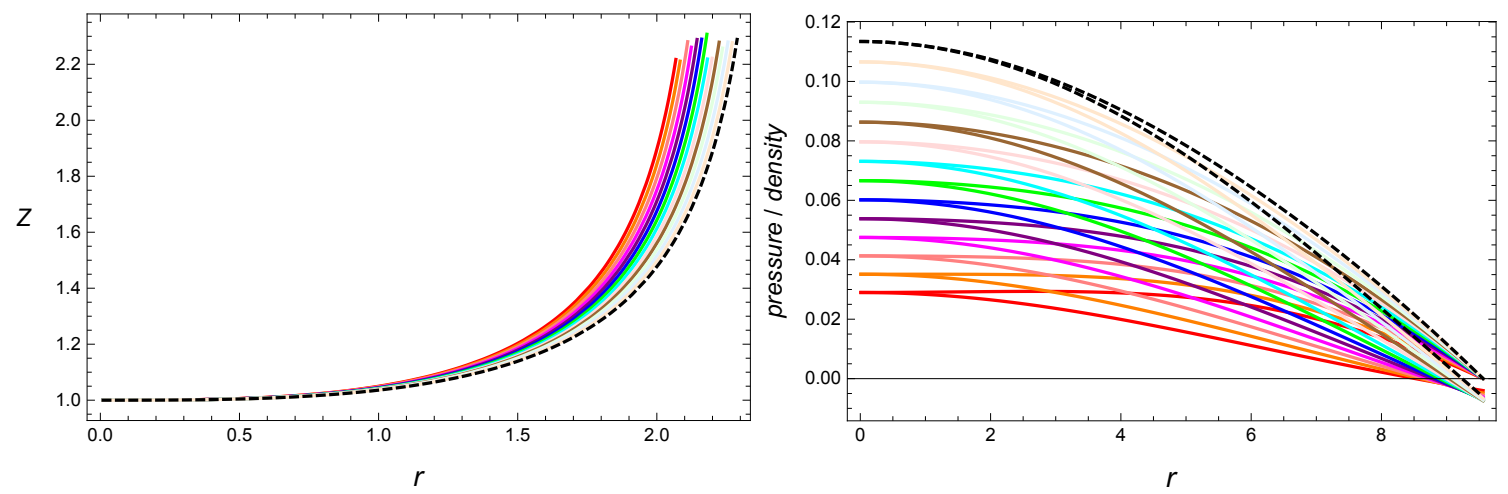

Figure 20. Plots correspond to the behavior of surface red-shift function (left) and equation of state (EoS) parameters (right) versus $r$.
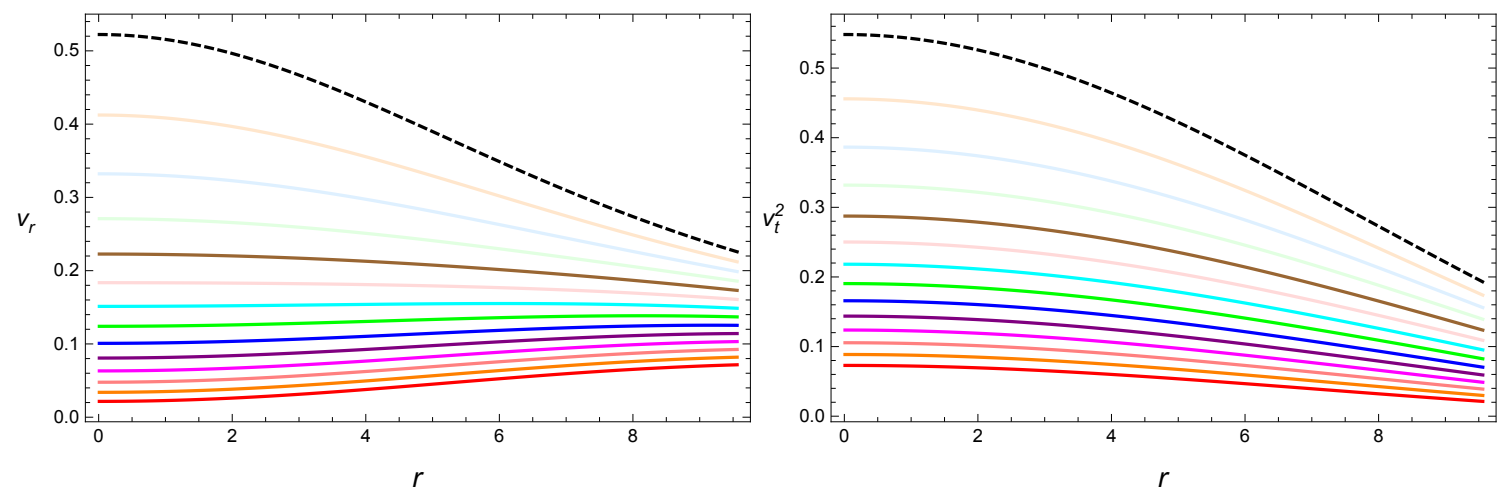

Figure 21. Shows the graphical response of $v_{r}^{2}$ (left) and, $v_{t}^{2}$ (right) against $r$.
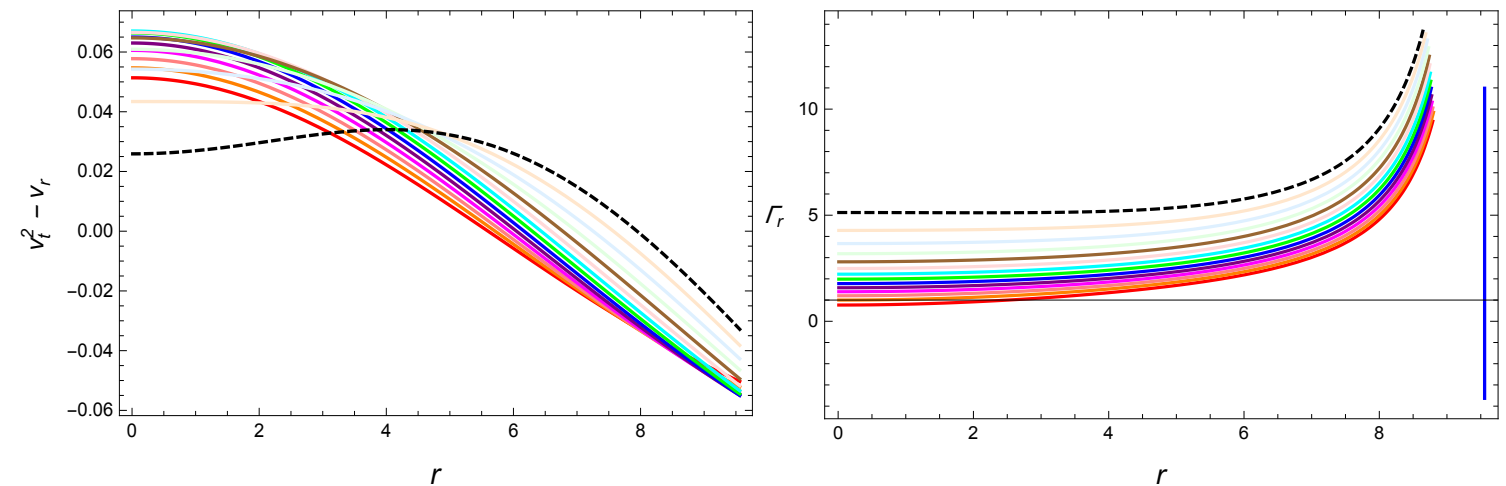

Figure 22. Shows the behavior of $v_{t}^{2}-v_{r}^{2}$ (left) and $\Gamma_{r}$ (right) against $r$. 
Table 3. Calculated values of $c, A$ and $B$ under Vela $X-1\left(1.77 M_{\odot}, 9.56 \mathrm{~km}\right)$ with $a=0.001 \mathrm{~km}^{-2}$ and $b=0.0015 \mathrm{~km}^{-2}$ for different suitable values of $n$ with $\lambda=2$.

\begin{tabular}{ccccc}
\hline $\mathbf{n}$ & $\mathbf{c}$ & $\mathbf{A}$ & $\mathbf{B}$ & $p_{r c} / \rho_{c}$ \\
\hline 1.8 & 0.00710 & 4.870026 & 0.065561 & 9.2046 \\
\hline 2.2 & 0.00686 & -6.929210 & 0.066135 & 9.5856 \\
\hline 2.6 & 0.00662 & -2.519258 & 0.066720 & 9.9767 \\
\hline 3.0 & 0.00639 & -1.391169 & 0.067316 & 10.3777 \\
\hline 3.4 & 0.00617 & -0.624852 & 0.067922 & 10.7924 \\
\hline 3.8 & 0.00596 & 0.998392 & 0.068539 & 11.2200 \\
\hline 4.2 & 0.00576 & -2.674653 & 0.069167 & 11.6628 \\
\hline 4.6 & $0.00556 ;$ & -1.187836 & 0.069807 & 12.1187 \\
\hline 5.0 & 0.00537 & -0.761750 & 0.070459 & 12.5898 \\
\hline 5.4 & 0.00518 & -0.460970 & 0.071123 & 13.0764 \\
\hline 5.8 & 0.00500 & 0.071800 & 0.106334 & 13.5785 \\
\hline 6.2 & 0.00483 & -1.027555 & 0.072490 & 14.0983 \\
\hline 6.6 & 0.00467 & -0.515308 & 0.073193 & 14.6350 \\
\hline 7.0 & 0.00451 & -0.346781 & 0.073911 & 15.1897 \\
\hline
\end{tabular}

The graphical behavior of energy constraints is provided in Figures 17 and 18 which showed that these constraints are valid for the present compact star model. The curves for mass function, compactness parameter and surface red-shift function are provided in Figures 19 and 20, which indicate positive increasing behavior and hence favors to the physically acceptable model of compact star. Figures 21 and 22 provide the graphical response of speed of sound parameters (radial and tangential) as well as adiabatic index. It can be easily observed that for large values of $\lambda$, the conditions $-1<v_{t}^{2}-v_{r}^{2}<0$ and $\Gamma_{r}>\frac{4}{3}$ fail to hold and hence refer to a non-realistic compact star model.

\section{Conclusions}

In the present paper, we have investigated a new family of solutions of embedding class-I model to find the physically acceptable solutions for Vela $X-1$ compact star in modified $f(\mathscr{R}, \mathcal{T})$ theory of gravity. For this purpose, we have adopted the well-known Karmarkar condition in the reference of Pandey-Sharma condition. Herein this study, we have explored some interesting physical properties related to the compact star configuration graphically and analytically. For the present study, we have taken spherically symmetric geometry with the matter contents involving anisotropic pressure profile without any net electric charge. Here we have assumed $e^{v(r)}=1+\frac{\left(a r^{2}+1\right)^{n} c r^{2}}{\left(b r^{2}+1\right)^{2}}$ model for $g_{r r}$ metric component. It is seen that the parameter $n$ has an important role in the discussion as the the compact star solutions for the choices $n=2, n=4, n=6$ can not be calculated. It is also observed that some important physical properties are also not satisfied for $n=7$. Likewise, it is found that the solutions for $n>7$ can not be calculated. In addition, we have used a simple linear model given by $f(\mathscr{R}, \mathcal{T})=\mathscr{R}+\lambda \mathcal{T}$ for this study. In this model, the parameter $\lambda$ has also an important role for the present discussion. In order to check its impact on the obtained solution, we have considered its two values given by $\lambda=0.35$ and $\lambda=2$. It is seen that the obtained solutions exhibited stable and realistic behavior for $n \in[1.8,7)-2,4,6$. For $\lambda=0.35$, the graphical illustration of different physical properties have been presented in Figures 1-11. In the following, we shall summarize our major findings.

- From Figure 1, it is seen that the metric components, i.e., $g_{t t}=e^{\mu}$, and $g_{r r}=e^{v}$, are calculated as $e^{\nu(r=0)}=1$ and $e^{\mu(r=0)} \neq 0$, which shows that this model is physically realistic and acceptable for Vela $X-1$ compact star; 
- The Zeldovich's condition, i.e., $p_{r c} / \rho_{c} \leq 1$, has been observed to be satisfied which can be confirmed From the Table 1;

- The energy density function has remained positive throughout in the stellar interior Vela $X-1$ compact object for this current model which can be seen from Figure 1;

- Both the radial and tangential pressure components has remained positive throughout in the stellar interior for Vela $X-1$ compact star. This behavior of both components can be verified from Figure 2;

- It is found that the measure of anisotropic pressure $\triangle(r)$ is positive throughout the region of the compact star and consequently, it supports to the structure of compact star which has been provided in Figure 3;

- $\quad$ The derivative of energy density function and radial pressure with respect to radial coordinate are calculated as negative and it is also confirmed form their graphical behavior given in Figure 3;

- $\quad$ From the Table 2 and Figures 4 and 5, it is found that the forces $\mathscr{F}_{\mathrm{a}}, \mathscr{F}_{\mathrm{h}}$ and $\mathscr{F}_{\mathrm{g}}$ are consistent with the equilibrium Condition for Vela $X-1$ model. It is also observed that these forces balanced each other's effect and hence left the whole configuration stable;

- $\quad$ The energy conditions, i.e., $\rho, p_{r}, p_{t}, \rho-p_{r}, \rho-p_{t}, \rho-p_{r}-2 p_{t}$, are observed to be satisfied for this model. Their graphically illustration has been provided in Figures 6 and 7;

- The mass-radii function $m(r)$ has remained positive, increasing and regular. Its graphical behavior can be observed from Figure 8;

- $\quad$ The compactness parameter $u(r)$ has remained positive and it also satisfied the Buchdahl limit, i.e., $u(r) \leq 8 / 9$ which can be seen from Figure 8;

- $\quad$ From the graph, it is observed that the surface red-shift function, i.e., $Z_{S}$ turned to be zero at $r=0$ and gradually increased with the increasing radial coordinate. In addition, it satisfied the Bohmer and Harko condition under the anisotropic configuration, i.e., $Z_{S}<5$;

- In radial and tangential directions, we have defined two equation of state (EoS), namely $w_{r}$ and $w_{t}$. It is noticed that the values of these EoS parameters remained positive inside the stellar object and also both assumed values less than 1 which can be seen from Figure 9;

- It is expressed From the Table 2 and Figures 10 and 11 that the radial and tangential speeds of sound for compact stars, which are denoted by $v_{r}^{2}$ and $v_{t}^{2}$ satisfied the necessary condition for $1.8 \leq n<7$, while for $n=7$, these radial and tangential velocities are observed greater than 1 , and consequently, violated the necessary condition. Further, the causality stability condition for $n=7$ is also violated for the present model;

- In reference of adiabatic index, it is found From the Table 2 and Figure 11 that the adiabatic index $\Gamma_{r}$ satisfied the inequality $\Gamma_{r}>4 / 3$ and showed increasing behavior.

For the second choice $\lambda=2.00$, the following properties are failed to meet the basic criteria for the existence of realistic compact stars.

- $\quad$ Tangential pressure profile has turned out as negative near the boundary, i.e., $r=R$ of astral objects. The negative tangential pressure components showed that our calculated solutions are non-realistic.

- The anisotropy function exhibited negative behavior throughout the configuration. The non-zero anisotropy function is a necessary requirement for the existence of realistic objects.

- The expression $v_{t}^{2}-v_{r}^{2}$ turned out to be inconsistent with the Abrea condition, i.e., $-1 \leq v_{t}^{2}-v_{r}^{2} \leq 0$.

- $\quad$ Likewise, the Adiabatic index, i.e., $\Gamma_{r}$ has also been incompatible with the condition $\Gamma_{r}>4 / 3$.

It is found that these solutions fulfill all the necessary conditions under the observational radii and mass attribute data for small values of parameter $\lambda=0.35$ and hence physically well-behaved and promising. The graphical analysis of different properties of our obtained solution is given in Figures 12-22 for $\lambda=2$. In fact, the obtained solutions are not confirmed satisfied entirely for $\lambda>0.35$. It is not possible to define a limit on parameter $\lambda$ with observational data in this present study. The smaller values of $\lambda$ have provided us a well-behaved results with good accuracy. Hence, to sum-up, it can be concluded that our obtained model exhibited well-behaved nature and physically considerable 
for $1.8 \leq n<7$. For other choices of parameter $n$ and $\lambda$, the solutions do not correspond to a realistic compact star model. If we put $\lambda=0$, we can get the results for GR case, which is already discussed by Singh et al. [53].

Author Contributions: Conceptualization, S.W. and M.Z.; methodology, G.M., S.W., and A.A.; software, G.M. and A.A.; validation, S.W., G.M., M.Z., and A.A.; formal analysis, S.W. and M.Z.; investigation, S.W., G.M., M.Z., and A.A.; data curation, S.W. and G.M.; writing —original draft preparation, S.W. , A.A., and G.M.; writing—review and editing, M.Z.; visualization, M.Z.; supervision, S.W. and M.Z. All authors have read and agreed to the published version of the manuscript.

Funding: This research has received APC fee from Prince Mohammad Bin Fahd University, Al Khobar 31952, Saudi Arabia.

Acknowledgments: We are grateful to the anonymous referees for their valuable comments, which improved the presentation of this paper. Saira Waheed thanks to Prince Mohammad Bin Fahd University, Al Khobar 31952, Saudi Arabia for financial support.

Conflicts of Interest: The authors declare no conflict of interest.

\section{References}

1. Schwarzschild, K. Über das Gravitationsfeld einer Kugel aus inkompressibler Flüssigkeit nach der Einsteinschen Theorie. Sitz. Dtsch. Akad. Wiss. Math. Phys. Berl. 1916, 24, 424.

2. Tolman, R.C. Static solutions of Einstein's field equations for spheres of fluid. Phys. Rev. 1939, 55, 364. [CrossRef]

3. Oppenheimer, J.R.; Volkoff, G.M. On massive neutron cores. Phys. Rev. 1939, 55, 374. [CrossRef]

4. Oppenheimer, J.R.; Snyder, H. On continued gravitational contraction. Phys. Rev. 1939, 56, 455. [CrossRef]

5. Jeans, J. The motions of stars in a Kapteyn universe. Mon. Not. R. Astron. Soc. 1922, 82, 122-132. [CrossRef]

6. Lemaitre, G. L'univers en expansion. Ann. Soc. Sci. Brux. A 1937, 10, 1-19.

7. Bowers, R.L.; Liang, E.P.T. Anisotropic spheres in general relativity. Astrophys. J. 1974, 188, 657. [CrossRef]

8. Ruderman, R. Pulsars: Structure and dynamics. Annu. Rev. Astron. Astrophys. 1972, 10, 427-476. [CrossRef]

9. Dev, K.; Gleiser, M. Anisotropic Stars: Exact Solutions. Gen. Relat. Gravit. 2002, 34, 1793-1818. [CrossRef]

10. Herrera, L.; Santos, N.O. Local anisotropy in self-gravitating systems. Phys. Rep. 1997, 286, 53-130. [CrossRef]

11. Riess, A.G.; Filippenko, A.V.; Challis, P.; Clocchiatti, A.; Diercks, A.; Garnavich, P.M.; Gilliland, R.L.; Hogan, C.J.; Jha, S.; Kirshner, R.P.; et al. Observational evidence from supernovae for an accelerating universe and a cosmological constant. Astron. J. 1998, 116, 1009. [CrossRef]

12. Perlmutter, S.; Aldering, G.; Goldhaber, G.; Knop, R.A.; Nugent, P.; Castro, P.G.; Deustua, S.; Fabbro, S.; Goobar, A.; Groom, D.E.; et al. Measurements of $\Omega$ and $\Lambda$ from 42 high-redshift supernovae. Astrophys. J. 1999, 517, 565. [CrossRef]

13. de Bernardis, P.; Ade, P.A.; Bock, J.J.; Bond, J.R.; Borrill, J.; Boscaleri, A.; Coble, K.; Crill, B.P.; De Gasperis, G.; Farese, P.C.; et al. A flat Universe from high-resolution maps of the cosmic microwave background radiation. Nature 2000, 6781, 955-959. [CrossRef] [PubMed]

14. Knop, R.A.; Aldering, G.; Amanullah, R.; Astier, P.; Blanc, G.; Burns, M.S.; Conley, A.; Deustua, S.E.; Doi, M.; Ellis, R.; et al. New constraints on $\Omega \mathrm{M}, \Omega \Lambda$, and w from an independent set of 11 high-redshift supernovae observed with the Hubble Space Telescope. Astrophys. J. 2003, 598, 102. [CrossRef]

15. Capozziello, S.; De Laurentis, M. Extended theories of gravity. Phys. Rep. 2011, 509, 167-321. [CrossRef]

16. Capozziello, S.; De Laurentis, M. The dark matter problem from $f(\mathscr{R}, \mathcal{T})$ gravity viewpoint. Ann. Phys. 2012, 524, 545-578. [CrossRef]

17. Starobinksy, A.A. A New Type of Isotropic Cosmological Models Without Singularity. Phys. Lett. B 1980, 91, 99-102. [CrossRef]

18. Bhawal, B.; Kar, S. Lorentzian wormholes in Einstein-Gauss-Bonnet theory. Phys. Rev. D 1992, 46, 2464. [CrossRef]

19. Rahaman, F.; Kalam, M.; Ghosh, A. Existence of wormholes in Einstein-Kalb-Ramond space time. arxiv 2006, arxiv:gr-qc/0605095.

20. Chakraborty, S; Bandyopadhyay, T. Wormhole and its analogue in brane world. arXiv 2008, arXiv:0707.1181.

21. Ferraro, R.; Fiorini, F. Modified teleparallel gravity: Inflation without an inflaton. Phys. Rev. D 2007, 75, 084031. [CrossRef] 
22. Sharif, M.; Waheed, S. Energy conditions in a generalized second-order scalar-tensor gravity. Adv. High Energy Phys. 2013, 2013. [CrossRef]

23. Kofinas, G.; Saridakis, N.E. Teleparallel equivalent of Gauss-Bonnet gravity and its modifications. Phys. Rev. D 2014, 90, 084044. [CrossRef]

24. Zubair, M.; Kousar, F. Cosmological reconstruction and energy bounds in $f\left(R, R_{\alpha \beta} R^{\alpha \beta}, \phi\right)$ gravity. Eur. Phys. J. C 2016, 76, 254. [CrossRef]

25. Ketov, S.V. Modified supergravity and early universe: The meeting point of cosmology and high-energy physics. Int. J. Mod. Phys. A 2013, 28, 1330021. [CrossRef]

26. Harko, T.; Lobo, F.S.N.; Nojiri, S.; Odintsov, S.D. $f(\mathscr{R}, \mathcal{T})$ gravity. Phys. Rev. D. 2011, 84, 024020. [CrossRef]

27. Jamil, M.; Momeni, D.; Myrzakulov, R. Attractor solutions in $f(\mathscr{R}, \mathcal{T})$ cosmology. Eur. Phys. J. C. 2012, 72, 1959. [CrossRef]

28. Jamil, M.; Momeni, D.; Myrzakulov, R. Violation of the First Law of Thermodynamics in $f(\mathscr{R}, \mathcal{T})$ Gravity. Chin. Phys. Lett. 2012, 29, 109801. [CrossRef]

29. Shabani, H.; Farhoudi, M. $f(\mathscr{R}, \mathcal{T})$ cosmological models in phase space. Phys. Rev. D 2013, 88, 044048. [CrossRef]

30. Shabani, H.; Farhoudi, M. Cosmological and solar system consequences of $f(\mathscr{R}, \mathcal{T})$ gravity models. Phys. Rev. D 2014, 90, 44031. [CrossRef]

31. Moraes, P.H.R.S. Cosmological solutions from induced matter model applied to $5 \mathrm{D}$ gravity and the shrinking of the extra coordinate. Eur. Phys. J. C 2015, 75, 168. [CrossRef]

32. Sharif, M.; Yousaf, Z. Dynamical analysis of self-gravitating stars in $f(\mathscr{R}, \mathcal{T})$ gravity. Astrophys. Space Sci. 2014, 354, 471-479. [CrossRef]

33. Zubair, M.; Abbas, G.; Noureen, I. Possible formation of compact stars in gravity. Astrophys. Space Sci. 2016, 361, 8. [CrossRef]

34. Alhamzawi, A.; Alhamzawi, R. Gravitational lensing by $f(\mathscr{R}, \mathcal{T})$ gravity Int. J. Mod. Phys. D 2016, 25, 1650020. [CrossRef]

35. Moraes, P.H.R.S.; Arbañil, J.D.; Malheiro, M. Stellar equilibrium configurations of compact stars in $f(R, T)$ theory of gravity. J. Cosmol. Astropart. Phys. 2016, 6, 5. [CrossRef]

36. Das, A.; Rahaman, F.; Guha, B.K.; Ray, S. Compact stars $f(\mathscr{R}, \mathcal{T})$ gravity. Eur. Phys. J. C 2016, 76, 654. [CrossRef]

37. Karmarkar, K.R. Gravitational metrics of spherical symmetry and class one. Proc. Indian Acad. Sci. A 1948, 27, 56. [CrossRef]

38. Komathiraj, K.; Maharaj, S.D. Classes of exact Einstein-Maxwell solutions. Gen. Relat. Gravit. 2007, 39, 2079-2093. [CrossRef]

39. Ivanov, B.V. Collapsing shear-free perfect fluid spheres with heat flow. Gen. Relat. Gravit. 2012, 44, $1835-1855$. [CrossRef]

40. Ivanov, B.V. A conformally flat realistic anisotropic model for a compact star. Eur. Phys. J. C 2018, 78, 332. [CrossRef]

41. Bhar, P.; Singh, K.N.; Pant, N. Compact star modeling with quadratic equation of state in Tolman VII space-time. Indian J. Phys. 2017, 91, 701-709. [CrossRef]

42. Bhar, P.; Singh, K.N.; Rahaman, F.; Pant, N.; Banerjee, S. A charged anisotropic well-behaved Adler-Finch-Skea solution satisfying Karmarkar condition. Int. J. Mod. Phys. D 2017, 26, 1750078. [CrossRef]

43. Maurya, S.K.; Gupta, Y.K.; Ray, S.; Deb, D. Generalised model for anisotropic compact stars. Eur. Phys. J. C 2016, 76, 693. [CrossRef]

44. Maurya, S.K.; Gupta, Y.K.; Smith, T.T.; Rahaman, F. A new exact anisotropic solution of embedding class one. Eur. Phys. J. A 2016, 52, 191. [CrossRef]

45. Maurya, S.K.; Gupta, Y.K.; Ray, S.; Deb, D. A new model for spherically symmetric charged compact stars of embedding class 1. Eur. Phys. J. C 2017, 77, 45. [CrossRef]

46. Singh, K.N.; Bhar, P; Pant, N. A new solution of embedding class I representing anisotropic fluid sphere in general relativity. Int. J. Mod. Phys. D 2016, 25, 1650099. [CrossRef]

47. Singh, K.N.; Pant, N.; Govender, A.M. Anisotropic compact stars in Karmarkar spacetime. Chin. Phys. C 2017, 41, 015103. [CrossRef]

48. Singh, K.N.; Pant, N. A family of well-behaved Karmarkar spacetime describing interior of relativistic stars. Astrophys. Space Sci. 2016, 361, 173. [CrossRef] 
49. Singh, K.N.; Bhar, P.; Pant, N. Solutions of the Einstein's field equations with anisotropic pressure compatible with cold star model. Astrophys. Space Sci. 2016, 361, 339. [CrossRef]

50. Rahaman, F.; Chakraborty, K.; Kuhfittig, P.K.F.; Shit, G.C.; Rahman, M. A new deterministic model of strange stars. Eur. Phys. J. C 2014, 74, 3126. [CrossRef]

51. Malaver, M. Strange Quark Star Model with Quadratic Equation of State. Math. Appl. 2014, 1, 9-15.

52. Herrera, L.; Barreto, W. General relativistic polytropes for anisotropic matter: The general formalism and applications. Phys. Rev. D 2013, 88, 084022. [CrossRef]

53. Mustafa, G.; Zubair, M., Waheed, S.; Xia, T. Realistic stellar anisotropic model satisfying Karmarker condition in $f(R, T)$ gravity. Phys. J. C 2020, 80, 26.

54. Singh, K.N.; Pant, N.; Tewari, N.; Aria, A.K. Embedded class solutions compatible for physical compact stars in general relativity. Eur. Phys. J. A 2018, 54, 77. [CrossRef]

55. Buchdahl, H.A. General relativistic fluid spheres. Phys. Rev. D 1959, 116, 1027. [CrossRef]

56. Bohmer, C.G.; Harko, T. Bounds on the basic physical parameters for anisotropic compact general relativistic objects. Class. Quantum Gravit. 2006, 23, 6479. [CrossRef]

57. Abreu, H.; Hernandez, H.; Nunez, L.A. Sound speeds, cracking and the stability of self-gravitating anisotropic compact objects. Class. Quantum Gravit. 2007, 24, 4631. [CrossRef]

(C) 2020 by the authors. Licensee MDPI, Basel, Switzerland. This article is an open access article distributed under the terms and conditions of the Creative Commons Attribution (CC BY) license (http:/ / creativecommons.org/licenses/by/4.0/). 